\title{
Molecular profiling of dilated cardiomyopathy that progresses to heart failure
}

Michael A. Burke, ${ }^{1,2}$ Stephen Chang, ${ }^{2}$ Hiroko Wakimoto, ${ }^{2,3}$ Joshua M. Gorham,, ${ }^{2}$ David A. Conner, ${ }^{2}$ Danos C. Christodoulou, ${ }^{2}$ Michael C. Parfenov, ${ }^{2}$ Steve R. DePalma, ${ }^{2}$ Seda Eminaga, ${ }^{2}$ Tetsuo Konno, ${ }^{2}$ Jonathan G. Seidman, ${ }^{2}$ and Christine E. Seidman ${ }^{1,2,4}$

'Cardiovascular Division, Department of Medicine, Brigham and Women's Hospital, Boston, Massachusetts, USA. ${ }^{2}$ Department of Genetics, Harvard Medical School, Boston, Massachusetts, USA. ${ }^{3}$ Department of Cardiology, Boston Children's Hospital and Harvard Medical School, Boston, Massachusetts, USA. ${ }^{4}$ Howard Hughes Medical Institute, Harvard Medical School, Boston, Massachusetts, USA.

Dilated cardiomyopathy (DCM) is defined by progressive functional and structural changes. We performed RNA-seq at different stages of disease to define molecular signaling in the progression from pre-DCM hearts to DCM and overt heart failure (HF) using a genetic model of DCM (phospholamban missense mutation, $\mathrm{PLN}^{\mathrm{RgC} /+}$ ). Pre-DCM hearts were phenotypically normal yet displayed proliferation of nonmyocytes ( $59 \%$ relative increase vs. WT, $\left.P=8 \times 10^{-4}\right)$ and activation of proinflammatory signaling with notable cardiomyocyte-specific induction of a subset of profibrotic cytokines including TCF $\beta 2$ and TCF $\beta 3$. These changes progressed through DCM and HF, resulting in substantial fibrosis (17.6\% of left ventricle [LV] vs. WT, $\left.P=6 \times 10^{-33}\right)$. Cardiomyocytes displayed a marked shift in metabolic gene transcription: downregulation of aerobic respiration and subsequent upregulation of glucose utilization, changes coincident with attenuated expression of PPAR $\alpha$ and PPAR $\gamma$ coactivators $-1 \alpha$ (PCC1 $\alpha$ ) and $-1 \beta$, and increased expression of the metabolic regulator T-box transcription factor 15 (Tbx15). Comparing DCM transcriptional profiles with those in hypertrophic cardiomyopathy (HCM) revealed similar and distinct molecular mechanisms. Our data suggest that cardiomyocyte-specific cytokine expression, early fibroblast activation, and the shift in metabolic gene expression are hallmarks of cardiomyopathy progression. Notably, key components of these profibrotic and metabolic networks were disease specific and distinguish DCM from HCM.

Authorship note: M.A. Burke and S. Chang contributed equally to this work.

Conflict of interest: J.G. Seidman and C.E. Seidman are founders and owns shares in Myokardia Inc., a startup company that is developing therapeutics that target the sarcomere.

Submitted: February 4, 2016 Accepted: April 5, 2016 Published: May 5, 2016

Reference information: JCI Insight. 2016;1(6):e86898. doi:10.1172/jci.insight.86898.

\section{Introduction}

Dilated cardiomyopathy (DCM), a common cause of heart failure (HF), is a prevalent disorder that affects up to 1 in 250 individuals (1). DCM may arise as a primary genetic disorder or as a secondary manifestation of other cardiovascular or systemic conditions. Altered myocardial calcium homeostasis is a common feature in genetic and acquired forms of DCM and HF (2) and can perturb cardiac physiology by modulating contractile force, signaling pathways, and gene transcription.

To decipher the temporal expression of key molecular mediators and pathways in the progression to DCM and HF, we capitalized on a genetic mouse model that expresses a missense mutation (p.Arg9Cys) in phospholamban ( $\mathrm{PLN}^{\mathrm{R} 9 \mathrm{C} /+}$ ), a transmembrane phosphoprotein that inhibits the cardiac sarcoplasmic/ endoplasmic reticulum $\mathrm{Ca}^{2+}$-adenosine triphosphatase (SERCA2a) pump. Calcium cycling is abnormal in young $\mathrm{PLN}^{\mathrm{R} 9 \mathrm{C} /+}$ mice before the onset of overt cardiac remodeling (denoted as pre-DCM). However, $\mathrm{PLN}^{\mathrm{R} 9 \mathrm{C} /+}$ mice subsequently develop DCM that consistently progresses to fulminant $\mathrm{HF}$ and premature death, (3) thus recapitulating the chronologic manifestations in human patients with this mutation (3-5). To delineate the longitudinal consequences of altered calcium homeostasis in cardiac remodeling, we studied the transcriptional changes in cardiomyocytes and nonmyocytes from pre-DCM through DCM to HF.

Hypertrophic cardiomyopathy (HCM) is a genetic disorder caused by mutations in the protein constituents of the sarcomere. HCM shares pathophysiologic and histologic features with DCM, including dysregulated calcium homeostasis, ventricular remodeling, and increases in myocardial fibrosis; however, unlike DCM, progression to systolic dysfunction and end-stage disease is uncommon (6-8). We previously 
A 8 weeks

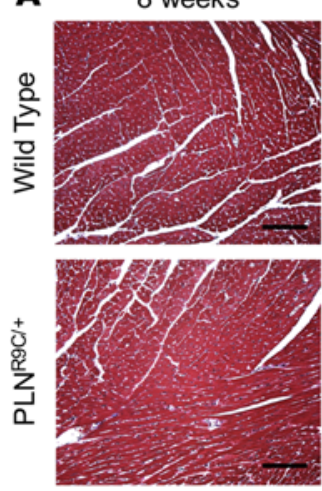

$\mathbf{B}$
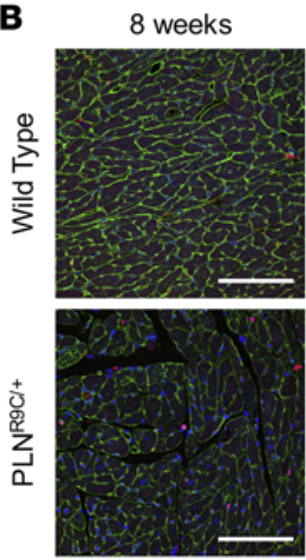

18 weeks

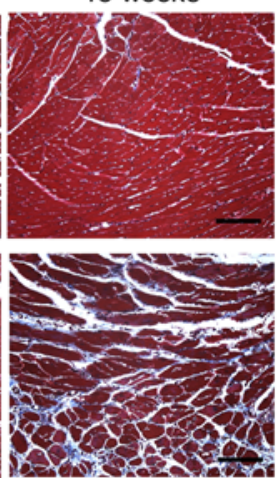

22 weeks
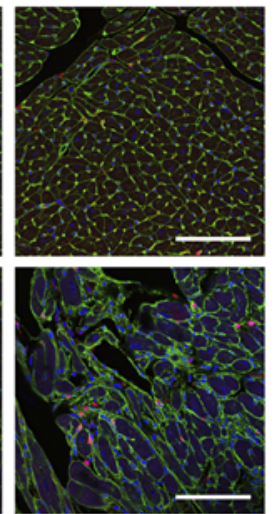

22 weeks

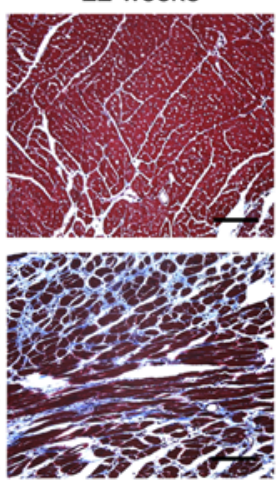

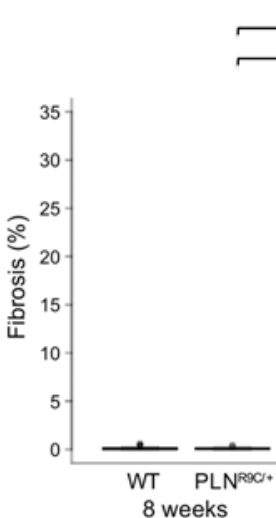
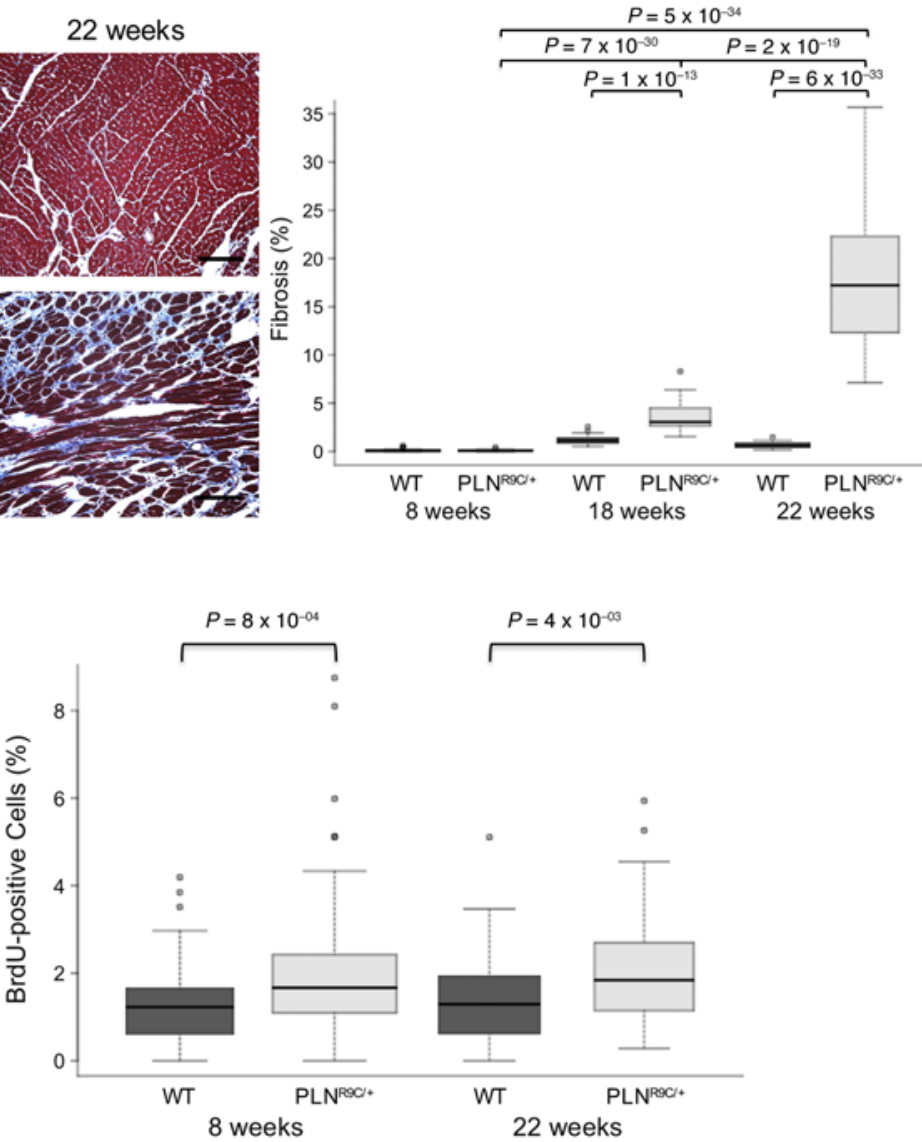

Figure 1. PLN ${ }^{\mathrm{RgC} /+}$ mice develop increased cardiac fibrosis and nonmyocyte cell proliferation with disease progression. (A) Light microscopy (scale bars: $100 \mu \mathrm{m}$ ) of Masson's trichrome-stained LV tissue at 8 weeks (pre-DCM), 18 weeks (DCM), and 22 weeks (overt HF) demonstrates progressive cardiac fibrosis in PLN ${ }^{\mathrm{Rg} /+}$ hearts compared with age-matched WT animals. Data quantitated were individual LV slices ( 2 per slide) at 10 levels (apex to base) from $n=3$ mice per group; 2-tailed Student's $t$ test. (B) Confocal microscopy (scale bars: $75 \mu \mathrm{m}$ ) and quantification of LV sections from hearts labeled with BrdU demonstrating nonmyocyte proliferation both pre-DCM and with overt HF. BrdU (magenta), wheat germ agglutinin (WGA; green), and nuclear DAPI (blue). Cells were counted from individual LV slices at 10 levels (apex to base) from $n=3$ mice per group. Greater than 25,000 nuclei were counted per experiment; 2-tailed Student's $t$ test.

reported transcriptional changes that occur in a mouse model with a human HCM mutation (p.Arg403Gln) in the myosin heavy chain gene $\operatorname{MHC}^{\mathrm{R} 403 \mathrm{C}}(9,10)$. Here, we compared the genes and pathways enriched in $\mathrm{PLN}^{\mathrm{R} 9 \mathrm{C} /+}$ mice with DCM to those enriched in $\mathrm{MHC}^{\mathrm{R} 403 \mathrm{Q}}$ mice with HCM. Our studies identified remarkable similarities and also key differences between these phenotypically distinct cardiomyopathies.

\section{Results}

Fibrosis and nonmyocyte proliferation characterize DCM in PLN ${ }^{\mathrm{RgC} /+}$ mice

We defined the temporal onset and progression of disease in $\mathrm{PLN}^{\mathrm{R} 9 \mathrm{C} /+}$ mice by echocardiography (Supplemental Table 1; supplemental material available online with this article; doi:10.1172/jci.insight.86898DS1) and cardiac histopathology. At 8 weeks of age, pre-DCM PLN ${ }^{\mathrm{R} 9 \mathrm{C} /+}$ mice had neither ventricular dilatation nor histopathologic findings of myocyte hypertrophy or cardiac fibrosis (Figure 1A and Supplemental Figures 1 and 2). Despite this, hearts from pre-DCM PLN ${ }^{\mathrm{R} 9 \mathrm{C} /+}$ mice displayed increased nonmyocyte BrdU labeling compared with WT controls $\left(2.0 \% \pm 1.5 \%\right.$ vs. $1.3 \% \pm 0.9 \% ; 59 \%$ relative increase; $P=8 \times 10^{-4}$, Figure 1B). By 18 weeks of age, overt DCM morphology had emerged, and histopathology showed myocyte enlargement (Supplemental Figure 2) and significant ventricular fibrosis $(3.6 \% \pm 1.5 \%$ vs. $1.1 \% \pm 0.4 \%$ WT, $\left.P=1 \times 10^{-13}\right)$. At approximately 22 weeks, $\mathrm{PLN}^{\mathrm{R} 9 \mathrm{C} /+}$ mice had manifested $\mathrm{HF}$ as evidenced by progressive upregulation of natriuretic peptides (Figure $2 \mathrm{~A})$ and weight loss $\left(\mathrm{PLN}^{\mathrm{R} 9 \mathrm{C} /+}\right.$ preterminal net weight change $-3.6 \pm 0.9 \mathrm{~g}$ vs. $0.7 \pm 0.8 \mathrm{~g}$ WT, $P=3 \times 10^{-8}$ ), consistent with cardiac cachexia (Supplemental Fig- 
A

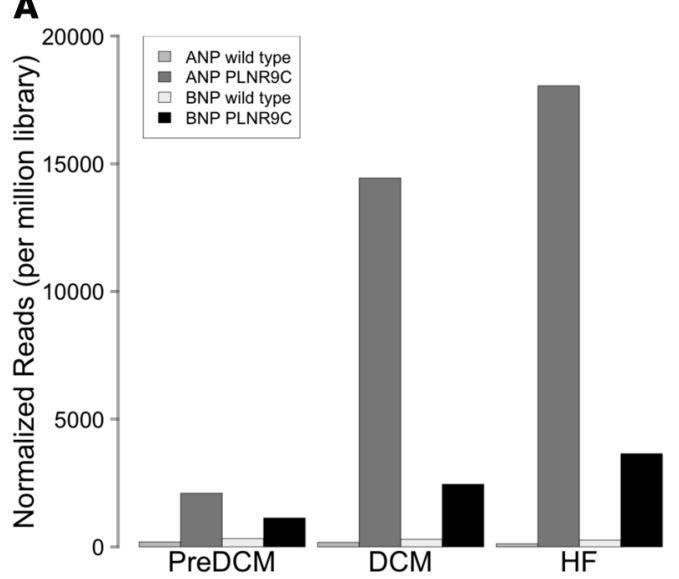

B

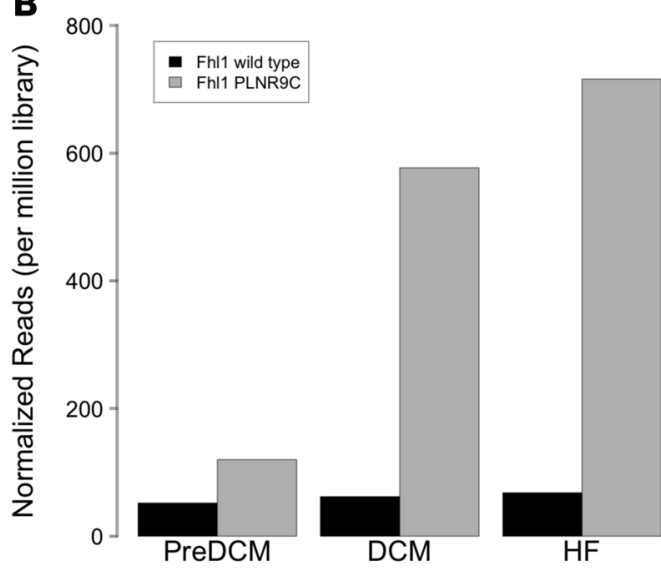

C

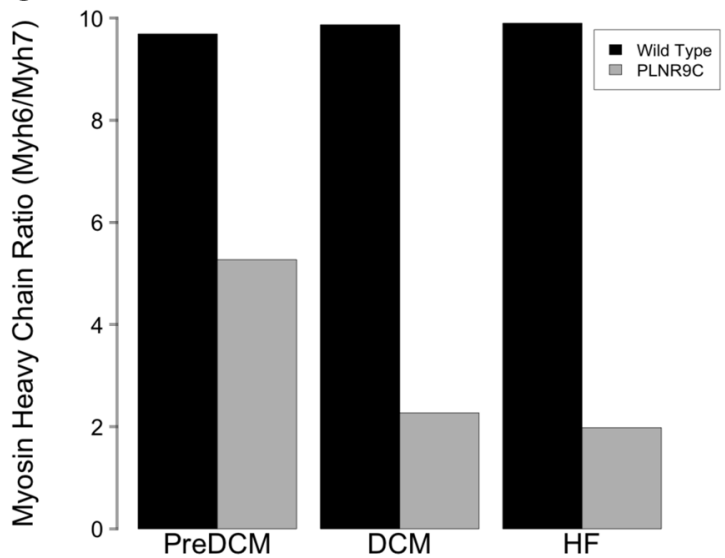

Figure 2. Activation of the cardiac stress response in PLN $^{\mathrm{RgC} /+}$ mice with disease progression. (A) Progressive and marked increase in atrial natriuretic peptide (ANP) and brain natriuretic peptide (BNP) mRNA levels with disease progression in $\mathrm{PLN}^{\mathrm{RgC} /+}$ mice $\left(P<1 \times 10^{-300}\right.$ vs. WT at all disease stages). (B) Progressive increase in 4-and-a-half LIM domains protein 1 (Fhl1) mRNA levels with disease progression in $\mathrm{PLN}^{\mathrm{RgC} /+}$ mice $\left(P<1 \times 10^{-300}\right.$ vs. WT at all disease stages). (C) Reduction in the ratio of adult ( $\alpha$-MHC/Myh6) to fetal ( $\beta$-MHC/Myh7) myosin heavy chain gene expression with disease progression in $\mathrm{PLN}^{\mathrm{RgC} /+}$ mice. RNA from $n=3$ mice was pooled prior to RNA-seq. Bayesian $P$ value corrected for multiple hypothesis testing (10).

ure 3). Mice also had notable behavioral changes, including lethargy with labored breathing and reduced peripheral perfusion, suggestive of low cardiac output. HF histopathology showed marked biventricular dilatation, cardiomyocyte hypertrophy, profound fibrosis $(17.6 \% \pm 6.9 \%$ vs $0.6 \% \pm 0.3 \%$ WT, $P=6 \times$ $\left.10^{-33}\right)$, and increased nonmyocyte BrdU staining $\left(2.0 \% \pm 1.2 \%\right.$ vs $1.4 \% \pm 1.0 \% \mathrm{WT}, P=4 \times 10^{-3}$; Figure 1 and Supplemental Figures 1 and 2). Based on BrdU labeling, we deduced that nonmyocyte proliferation contributed in part to increased gene transcription.

\section{RNA-seq reveals differences between myocyte and nonmyocyte gene expression}

Transcripts from left ventricle (LV) tissues derived from age-matched WT and $\mathrm{PLN}^{\mathrm{R} 9 \mathrm{C} /+}$ mice with preDCM, DCM, or HF were sequenced (RNA-seq) to identify genes with significantly altered expression (Supplemental Figure 4). We also performed RNA-seq on isolated cardiomyocytes and nonmyocytes from hearts of DCM and age-matched WT mice to categorize transcripts as predominantly expressed in either or both cell compartments. Separation of isolated cell populations was excellent, and RNA-seq data showed less than $4 \%$ cross-cell contamination of prototypic lineage-specific genes (Supplemental Table 2).

DCM mice had characteristic transcriptional changes indicating activation of the fetal gene program, which typifies HF. $\mathrm{PLN}^{\mathrm{R} 9 \mathrm{C} /+}$ mice displayed markedly increased expression of prototypical cardiomyocyte stress-response genes, including the natriuretic peptides ( $N p p a$ and $N p p b$ ) and 4-and-a-half LIM domains protein-1 (Fhl1), as well as a shift in myosin heavy chain gene expression from the adult (Myh) to fetal (Myh7) isoforms (Figure 2).

Nonmyocyte transcriptomes showed over 2,000 genes that were differentially expressed in pre-DCM compared with WT, and this gene set increased with the transition to DCM and HF (Table 1, Supplemental Table 3). Fully, 24\% $(n=1,209)$ of differentially expressed nonmyocyte genes were common to all stages (Figure 3). Prominent increases in expression of profibrotic cytokines such as TGF $\beta$ and connective tissue growth factor were observed, as were markers of extracellular matrix remodeling, including numerous 
Table 1. Differentially expressed genes in $\mathrm{PLN}^{\mathrm{RgC} /+}$ whole LV tissue

\begin{tabular}{|c|c|c|c|}
\hline \multicolumn{2}{|c|}{ Cell Population } & \multirow{2}{*}{$\begin{array}{l}\text { Differentially Expressed Genes } \\
\qquad 2,023\end{array}$} & \multirow{2}{*}{$\begin{array}{c}\text { Upregulated Genes }^{\mathrm{A}} \\
\text { 2,002 (99.0\%) }\end{array}$} \\
\hline Nonmyocytes & Pre-DCM & & \\
\hline & $\mathrm{HF}$ & 3,757 & 3,554 (94.6\%) \\
\hline \multirow[t]{2}{*}{ Myocytes } & Pre-DCM & 348 & 240 (69.0\%) \\
\hline & DCM & 1,069 & 285 (26.7\%) \\
\hline
\end{tabular}

APercent total differentially expressed genes.

collagen precursor genes, periostin, osteopontin, osteonectin (Sparc), tenascin-c, thrombospondin, matrix metalloproteinases, and metalloproteinase inhibitors (Supplemental Table 3). Notably, nonmyocytes had significantly increased expression of gene family members encoding complement factors, chemokines, and immune signaling receptors.

The cardiomyocyte transcriptome displayed a different dynamic pattern from that of nonmyocytes (Table 1 and Supplemental Table 3). Only 348 genes were differentially expressed in pre-DCM cardiomyocytes compared with WT, and only $12 \%$ of differentially expressed genes $\left(n=178 ; P=2 \times 10^{-16}\right)$ were common throughout all stages of disease (Figure 3 ). The majority (69\%) of pre-DCM differentially expressed cardiomyocyte genes were upregulated. Among these were genes that encode molecules involved in modulating calcium, including sarcolipin, sarcolemmal membrane-associated protein, calsequestrin, calreticulin, and the calcium-binding mitochondrial carrier. The onset of overt DCM marked a substantial shift and predominant downregulation in cardiomyocyte gene expression, including the expression of many genes encoding proteins that regulate cellular metabolic processes. With progression to HF, the overall cardiomyocyte transcriptome profile was largely unchanged, and while some transcripts varied, 54\% of differentially expressed cardiomyocyte genes were common to both DCM and HF (Figure 3).

\section{Divergent pathway activation in nonmyocytes vs. myocytes}

To provide a survey of the molecular processes altered in $\mathrm{PLN}^{\mathrm{R} 9 \mathrm{C} /+}$ nonmyocytes and cardiomyocytes, we used the bioinformatics tools Ingenuity pathway analysis (IPA) and gene ontology sequencing (GO-seq) to define canonical biological pathways, GO terms and Kyoto Encyclopedia of Genes and Genomics (KEGG) pathways at 3 different stages of disease (Supplemental Tables 4-6). Additionally, the Ingenuity upstream regulator analysis tool was used to predict the activation state of molecules associated with the pattern of differential gene expression at each stage of disease (see Methods for details).

Nonmyocytes. Enriched pathways in DCM nonmyocytes included those involved in translation, acute phase responses, integrin signaling, and cytokine signaling (Supplemental Tables 4-6) - processes that would promote inflammation and fibrosis. The pattern of differentially expressed nonmyocyte genes predicted the activation of all 3 TGF $\beta$ isoforms (Figure 4A). Expression of Tgfb2 (10.4-fold, $P=3 \times 10^{-277}$ ) and Tgfb3 (4.7-fold, $P=5 \times 10^{-177}$ ) was strongly increased in DCM hearts compared with WT (Figure 4B). By contrast, $T g f b 1$ expression was only modestly increased (1.9-fold, $P=2 \times 10^{-17}$ ), a finding likely related at least in part to the increased proliferation of nonmyocytes in $\mathrm{PLN}^{\mathrm{R} 9 \mathrm{C} /+}$ mice. RNA-seq of isolated DCM nonmyocytes revealed no change in Tgfb1 expression (1.05-fold, $P=0.06$ ). Quantitative PCR (qPCR) of TGF $\beta$ isoforms in a separate cohort $(n=24$ DCM and age-matched WT mice) confirmed selective activation of Tgfb2 (5.2-fold, $P=2 \times 10^{-8}$ ) and Tgfb3 (3.7-fold, $P=0.02$ ) but not Tgfb1 (1.4-fold, $P=0.10$ ). Consistent with these gene-expression data, all bioinformatics platforms identified enrichment of TGF $\beta$ signaling in $\mathrm{PLN}^{\mathrm{R} 9 \mathrm{C} /+}$ nonmyocytes.

We also observed increased expression of signaling molecules in $\mathrm{PLN}^{\mathrm{R} 9 \mathrm{C} /+}$ mice that would amplify TGF $\beta$-mediated fibrosis and inflammation. TGF $\beta$ receptors 1 and 2 (Tgfbr1, 2.7-fold, $P=3 \times 10^{-29}$; Tgfbr2, 2.4-fold, $P=7 \times 10^{-57}$ ) were significantly upregulated in DCM hearts, and with progression to HF, Tgfbr3 $\left(2.0\right.$-fold, $\left.1 \times 10^{-29}\right)$ was also increased. The TGFß1-responsive NADPH oxidase 4 (Nox4) gene, which mediates conversion of quiescent fibroblasts to active myofibroblasts, was also dramatically induced (56.5- 
A

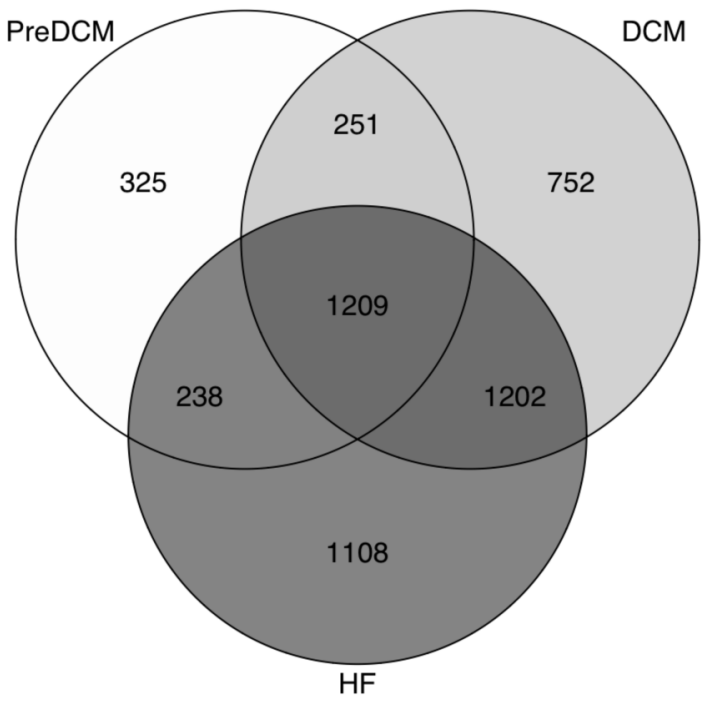

B

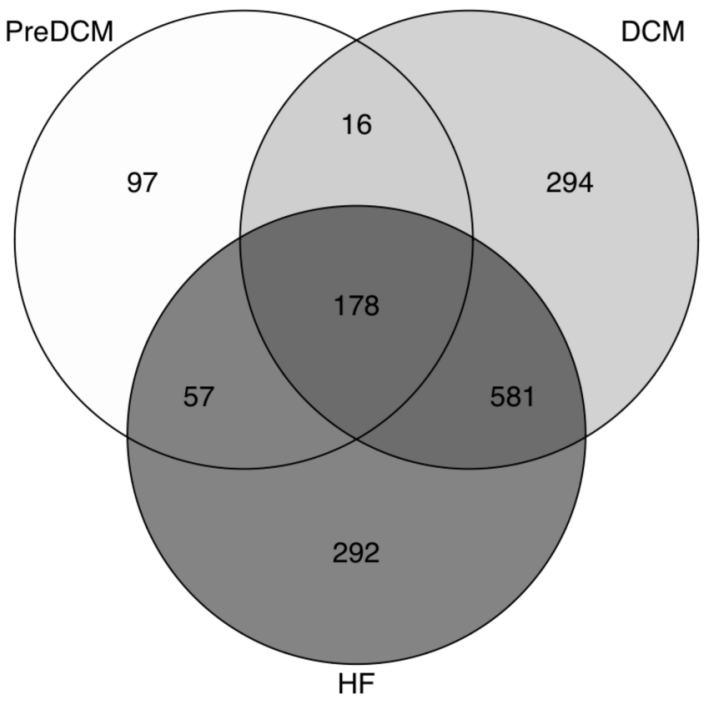

Figure 3. Differential gene expression at distinct stages of disease in nonmyocytes and myocytes from PLN ${ }^{\mathrm{RgC} /+}$ hearts. (A) Differences in nonmyocyte gene expression progress steadily with worsening disease in LV tissue, with nearly a quarter of differentially expressed genes common to all stages. (B) Few differentially expressed genes were unique to cardiomyocytes before DCM, and only $12 \%$ of differentially expressed genes were common to all stages. More significant changes were noted with onset of phenotypic disease.

fold, $2 \times 10^{-33}$ ) in DCM hearts (11). Additionally, a host of profibrotic transcription factors were upregulated in DCM nonmyocytes. Runt-related transcription factor 1 (Runx1, 4.8-fold, $P=4 \times 10^{-17}$ ) controls TGF $\beta$-dependent myofibroblast differentiation (12). The related molecule, Runx2 (4.3-fold, $\left.P=2 \times 10^{-9}\right)$, is a known regulator of collagen type-1 gene expression and was recently identified as a key mediator of vascular fibrosis (13). PU.1 (Sfpi1/Spi1, 3.3-fold, $P=3 \times 10^{-11}$ ) is a master transcription factor that regulates the cell-specific response to TGF $\beta$ signaling (14). Transcription factor 21 ( Tcf 21 , 3.2-fold, $P=3 \times 10^{-31}$ ) is highly expressed in regions of myocardial fibrosis (15). Though upstream control of this molecule is unclear, some evidence suggests Tcf 21 works in concert with the AP-1 transcription factor (16). JunB, a TGF $\beta$-responsive member of the AP-1 transcription factor family (17), is also upregulated in DCM hearts (2.4-fold, $P=1$ $\left.\times 10^{-26}\right)$. Also differentially expressed is E2F transcription factor $1\left(E 2 f 1,2.9\right.$-fold, $\left.P=3 \times 10^{-5}\right)$, which is known to play a role in the cardiac stress response (18) and is an important downstream effector of TGF $\beta$ in cancer and various fibrotic diseases $(19,20)$. The cyclic-AMP dependent transcription factor Atf3 (7.4fold, $P=2 \times 10^{-44}$ ), which promotes maladaptive cardiac remodeling and regulates cardiac fibrosis, was also upregulated in $\mathrm{PLN}^{\mathrm{R} 9 \mathrm{C} /+}(21,22)$.

$\mathrm{PLN}^{\mathrm{R} 9 \mathrm{C} /+}$ mice also displayed striking upregulation of the stress-response proto-oncogenes c-myc ( $M y c$, 8.7-fold, $P=2 \times 10^{-52}$ ) and c-fos (Fos, 11.2-fold, $P=8 \times 10^{-18}$ ) with DCM. Other developmental cardiac transcription factors, including Meox1 (6.1-fold, $\left.P=1 \times 10^{-131}\right)$, Sox17 (2.8-fold, $P=3 \times 10^{-18}$ ), (23) and Tbx20 (1.4-fold, $P=1.3 \times 10^{-8}$ ), (24) also had increased expression in $\mathrm{PLN}^{\mathrm{R} 9 \mathrm{C} /+}$ nonmyocytes, indicating that DCM incited a broad shift in transcriptional control of nonmyocytes.

Nonmyocytes from DCM hearts also had markedly augmented transcription of a number of components of the Wnt signaling network, which plays important roles in cardiac embryogenesis as well as remodeling in the adult heart after myocardial infarction and with $\mathrm{HF}$ (25). Notable among these were the frizzled receptors Fzd1 (2.2-fold, $P=1 \times 10^{-17}$ ), Fzd2 (3.3-fold, $P=3 \times 10^{-32}$ ), and Fzd7 (2.6-fold, $P=1 \times 10^{-19}$ ); the Wnt-signaling inhibitors-secreted frizzled-related protein-1 (Sfrp1, 7.3-fold, $P=3 \times 10^{-132}$ ); Sfrp2 (50.8-fold, $P=2 \times 10^{-174}$ ); and the Dickkopf Wnt signaling pathway inhibitor-3 ( $D k k 3,12.1$-fold, $\left.P=2 \times 10^{-77}\right)$. There was increased expression of the downstream effector WNT1-inducible signaling pathway protein-2 gene (Wisp2, 58.4-fold, $\left.P=3 \times 10^{-253}\right)$. Consistent with these data, pathway analyses identify enrichment of the canonical Wnt signaling pathway (Ingenuity pathway Wnt/ $\beta$-catenin pathway, $P=0.005$; GO term canonical Wnt receptor signaling pathway [GO:0060070], $P=0.0004)$ in the DCM nonmyocyte compartment. Both the increased proliferation of nonmyocytes and activation of inflammatory gene programs could account for the increased 
Table 2. Progressive enrichment of metabolism pathways in $\mathrm{PLN}^{\mathrm{RgC} /+}$ cardiomyocytes with DCM

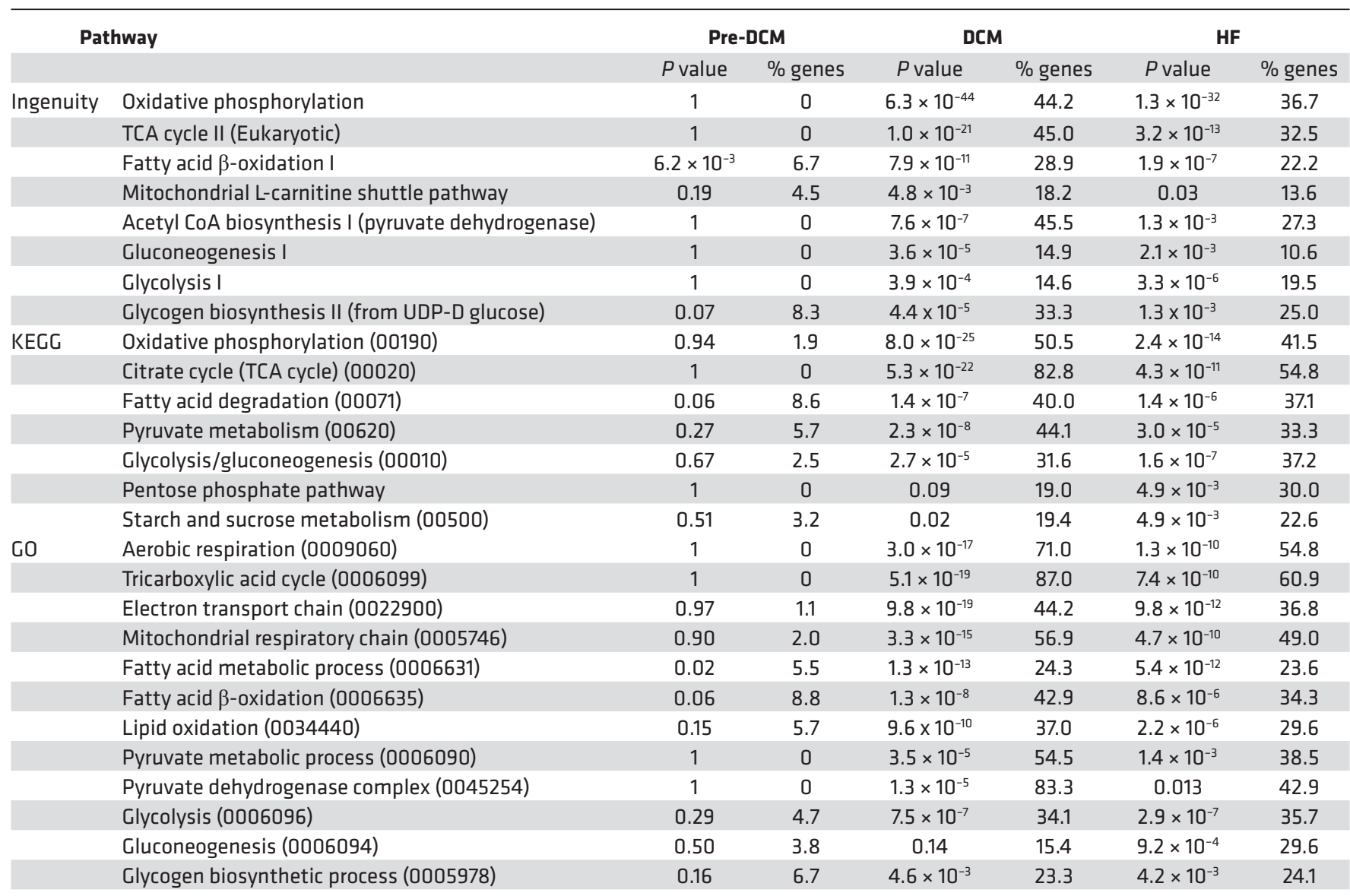

$\%$ genes, percentage of pathway genes that are differentially expressed in the data set.

burden of myocardial fibrosis that accompanied progressive ventricular remodeling in $\mathrm{PLN}^{\mathrm{R} 9 \mathrm{C} /+}$ mice.

Cardiomyocytes. DCM and HF cardiomyocytes exhibited transcriptional changes indicating altered cell signaling and cell metabolism. Although WT cardiomyocytes had little expression of TGF $\beta$ isoforms in comparison with nonmyocytes, isolated $\mathrm{PLN}^{\mathrm{R} 9 \mathrm{C} /+}$ cardiomyocytes had substantially increased expression of $T g f b 2$ (13.0-fold, $\left.P \leq 1 \times 10^{-300}\right)$ and $T g f b 3$ (4.5-fold, $\left.P=1 \times 10^{-58}\right)$. Tgfb1 levels ( -1.3 -fold, $P=0.001)$ were unchanged in DCM cardiomyocytes (Figure $4 \mathrm{C}$ ). Together, these data suggest there are distinct roles for the different TGF $\beta$ isoforms in cardiac remodeling. Interestingly, isolated cardiomyocytes from DCM mice also had increased expression of other proinflammatory cytokines, including growth/differentiation factor 15 ( $G d f 15,21.6$-fold, $\left.P<1 \times 10^{-300}\right)$ and connective tissue growth factor (Ctgf, 5.3-fold, $P<1 \times 10^{-300}$ ). Together, these findings suggest that cardiomyocytes have an active and dynamic role in profibrotic signaling in $\mathrm{HF}$.

With the onset of DCM, PLN ${ }^{\mathrm{R} 9 \mathrm{C} /+}$ mice displayed a nearly uniform reduction in expression of genes controlling aerobic metabolism, and as mice developed overt HF, there were continued gene expression changes, with progressive activation of genes regulating glucose metabolism (Figure 5). Consistent with these changes in gene expression, pathway analyses identified prominent reductions in aerobic metabolism (fatty acid oxidation, citric acid cycle, oxidative phosphorylation) and increased glucose metabolism (pyruvate metabolism, glycolysis, glycogen metabolism, gluconeogenesis; Supplemental Tables 4-6 and Table 2). Data were confirmed by $\mathrm{qPCR}$ for selected nuclear-encoded mitochondrial oxidative phosphorylation genes in a separate cohort of $12 \mathrm{PLN}^{\mathrm{R} 9 \mathrm{C} /+}$ mice with DCM or age-matched WT mice (Ndufs7-1.9-fold, $5 \times$ 10-5; Cox7a1-2.2-fold, $2 \times 10^{-4}$; Atp5a1-1.8-fold, $\left.2 \times 10^{-4}\right)$.

In addition to a shift from aerobic metabolism to glucose utilization, there was evidence for an 

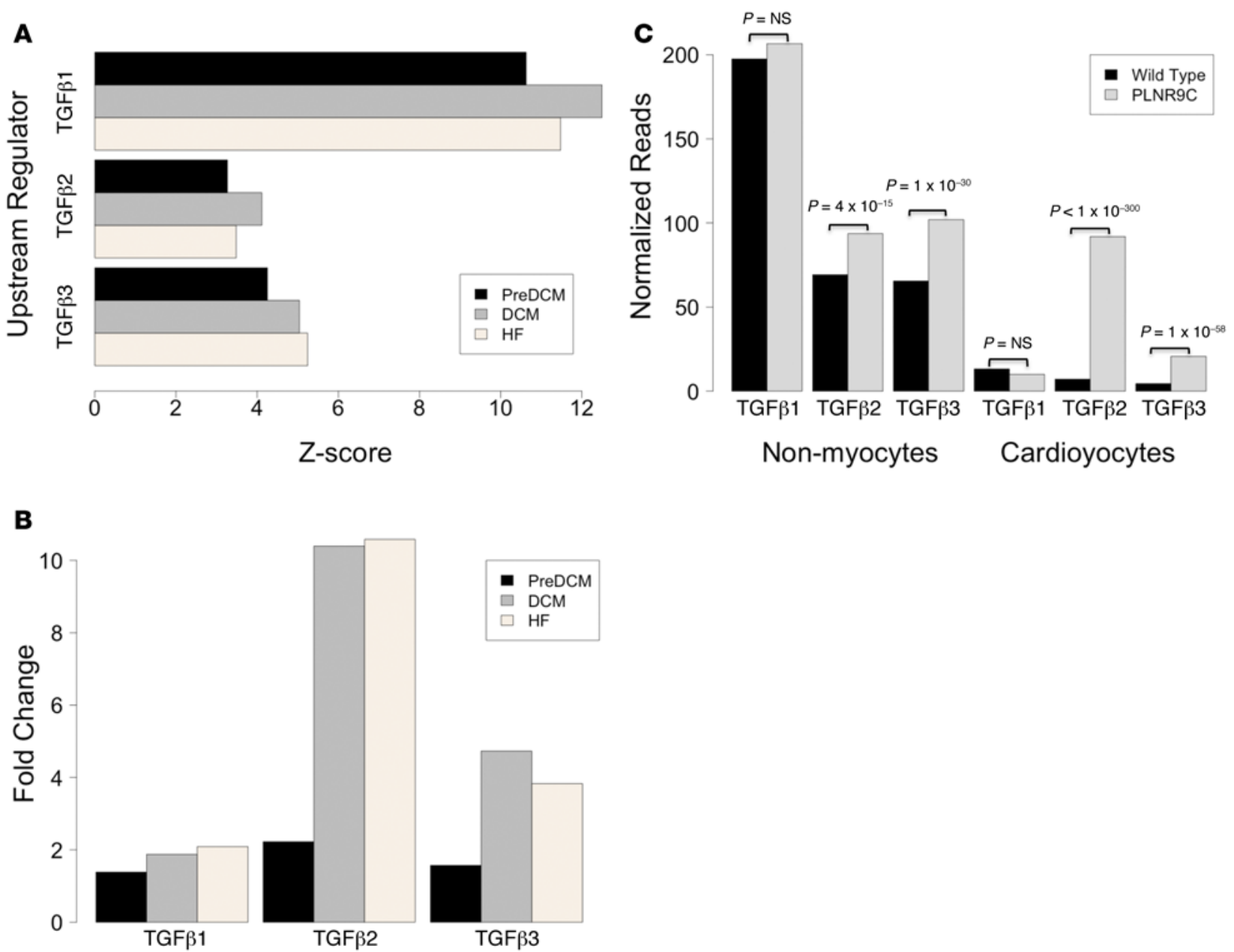

Figure 4. Increased expression of TGF $\beta$ in PLN ${ }^{\mathrm{RgC} /+}$ mice. (A) All TGF $\beta$ isoforms were predicted to be activated at all 3 stages of disease by upstream regulator analysis. Z-score reflects both the confidence and direction of the inferred activation state $\left(P<1 \times 10^{-6}\right.$ for all analyses). (B) TCF $\beta$ gene expression was induced in PLN ${ }^{R g C /+} \mathrm{LV}$ tissue at all stages of disease $(n=3$ mice pooled prior to RNA-seq). (C) Isolated nonmyocyte and cardiomyocyte cells ( $n=6$ mice pooled prior to RNA-seq) at 18 weeks (DCM) showed that TCF $\beta$ genes were predominantly expressed in WT nonmyocytes, with modest increases in Tgfb2 and Tgfb3 expression in PLN ${ }^{\mathrm{RgC} /+}$ nonmyocytes. In contrast, Tgfb2 and Tgfb3 were strongly induced in cardiomyocytes of PLN ${ }^{\mathrm{RgC/+}}$ mice with DCM. Tgfb1 levels do not change significantly in either cell compartment. Bayesian $P$ value corrected for multiple hypothesis testing (10).

increase in nonoxidative glucose metabolism. Key genes in the production of N-acetyl-glucosamine by the hexosamine biosynthetic pathway were upregulated in isolated cardiomyocytes (Gfpt1 1.7-fold, $P=2$ $\times 10^{-7}$; Gnpnat1 1.5-fold, $P=3 \times 10^{-4}$; Pgm3 1.7-fold, $P=1 \times 10^{-3}$ ), though it is noted that expression of the genes encoding the enzymes controlling protein O-glycosylation (Ogt, Mgea5) were not altered with the development of DCM or HF. Also enriched were the pentose-phosphate pathway and glycogen biosynthesis (Table 2).

The cardiomyocyte transcriptional changes in DCM and HF hearts predicted substantive changes in metabolic regulators, such as inhibition of signaling by the PPAR pathway (Figure 6A), including the master metabolic regulator Ppary coactivator $1 \alpha$ (Ppargc1a). Indeed, the expression of many PPAR genes and key cofactors were significantly reduced with development of DCM and HF (Figure 6B). Data were confirmed by qPCR for selected PPAR family members in a separate cohort of $12 \mathrm{PLN}^{\mathrm{R} 9 \mathrm{C} /+}$ mice with DCM or age-matched WT mice (Ppara-3-fold, $P=8 \times 10^{-4}$; Ppargc1a-3.9-fold, $P=4 \times 10^{-3}$; Ppargclb-2.0-fold, $\left.P=9 \times 10^{-4}\right)$. Metabolic pathways affected by PPAR signaling match those that are enriched in PLN ${ }^{\mathrm{R} 9 \mathrm{C} /+}$ mice with DCM and HF (Figure 6C).

In addition to these well-described metabolic regulators, we also identified increased expression of T-box transcription factor 15 (Tbx15) in DCM hearts (8.5-fold, $\left.P=1 \times 10^{-15}\right)$. Interestingly, induction was exclusive to cardiomyocytes (11.7-fold, $P=8 \times 10^{-27}$ in isolated cardiomyocytes, with no detectable expression in iso- 
A

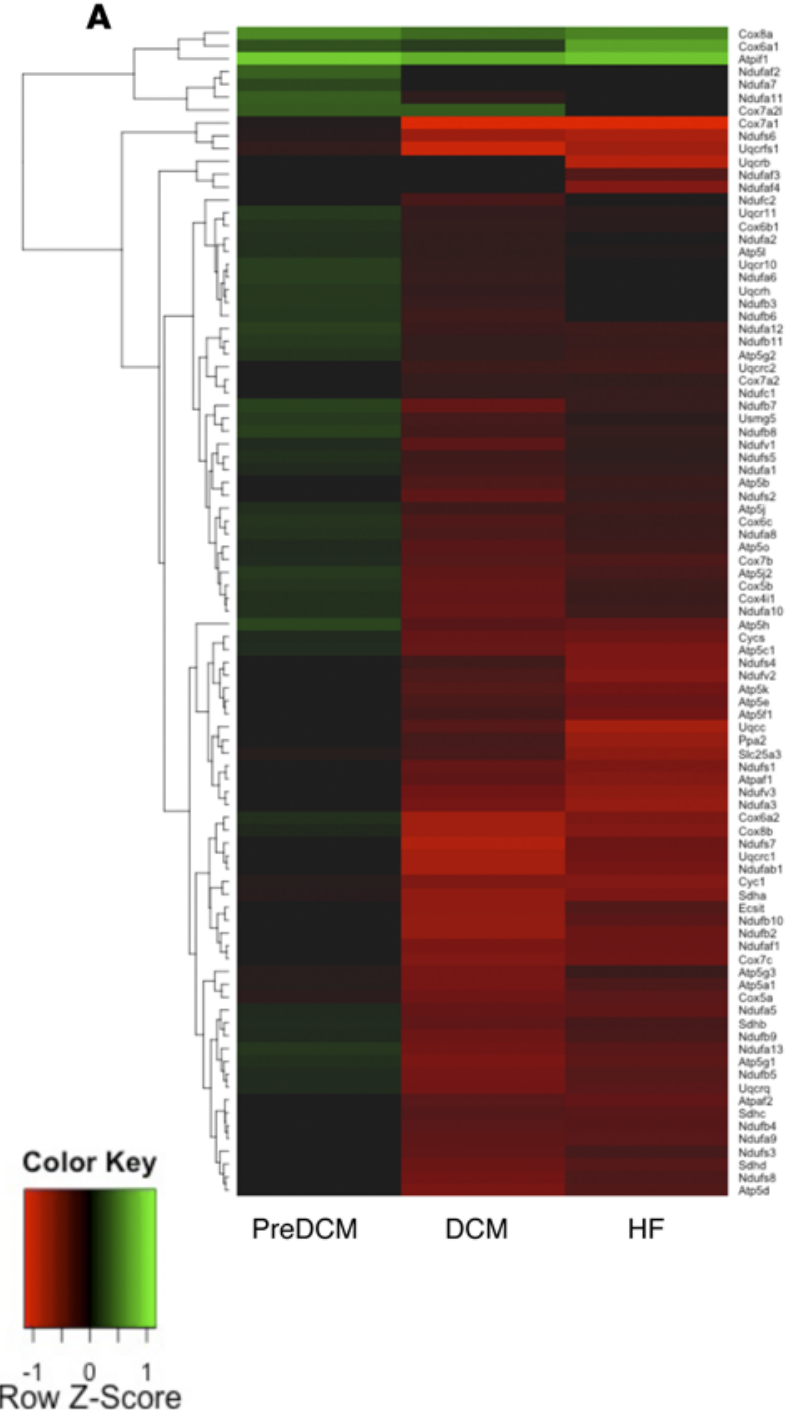

B

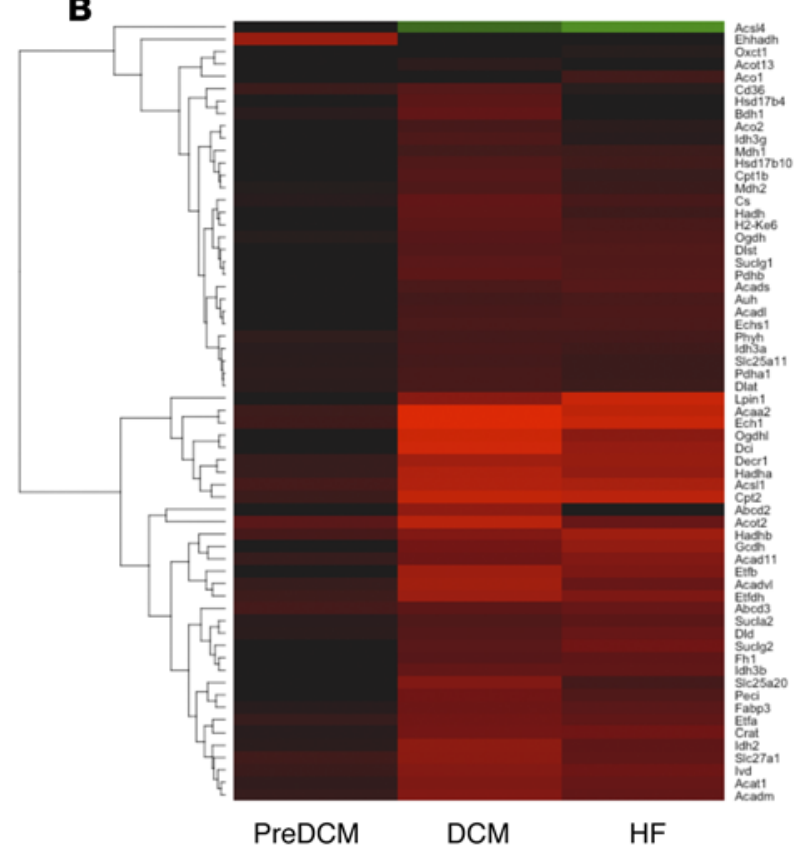

C

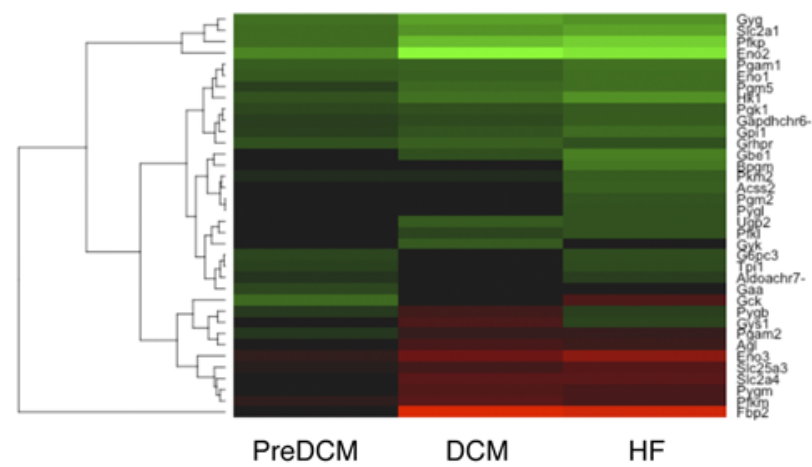

Figure 5. Heat maps of metabolic genes demonstrate marked dysregulation of myocyte metabolism. (A) Mitochondrial genes controlling oxidative phosphorylation were significantly downregulated with the onset of DCM. (B) There was also reduced expression of genes governing fatty acid $\beta$ oxidation and the citric acid cycle with the onset of DCM. (C) By contrast, genes associated with glucose utilization became dysregulated with the onset of DCM, and their expression was progressively increased with overt HF. Data plotted are fold-change values versus age-matched WT controls $(n=3$ mice pooled prior to RNA-seq).

lated nonmyocytes). Data were confirmed by qPCR in a separate cohort of $12 \mathrm{PLN}^{\mathrm{R} 9 \mathrm{C} /+}$ mice with DCM or age-matched WT mice ( 8.5 -fold, $P=1 \times 10^{-3}$ ). In addition to its developmental functions, Tbx15 has been implicated in the regulation of glycolytic metabolism in skeletal muscle fibers (26). We suggest that the complementary activation of Tbx15 drives the increase in glycolytic metabolism in conjunction with the inhibition of PPAR signaling; together, this results in the profound metabolic changes that characterize DCM and HF.

\section{Common and distinct pathways emerge in DCM and HCM}

Using previously reported differential gene expression in a genetic mouse model of HCM (MHC ${ }^{\mathrm{R} 403 \mathrm{O}}$ ) (10), we performed pathway analysis using the bioinformatics platforms described herein to define canonical pathways, GO terms, KEGG pathways, and upstream regulators in HCM nonmyocytes and cardiomyocytes, and we compared these with DCM datasets from $\mathrm{PLN}^{\mathrm{R} 9 \mathrm{C} /+}$ mice. HCM nonmyocytes were enriched in profibrotic and inflammatory/immune pathways while HCM cardiomyocytes were notable for substantial changes in metabolism (Figure 7A and Supplemental Table 7), patterns that were remarkably similar to those observed in DCM mice. Despite these similarities, the majority of differentially expressed genes were unique to one or the other cardiomyopathy (Figure 7B). Among nonmyocyte genes with differential expression, 39\% were common and 

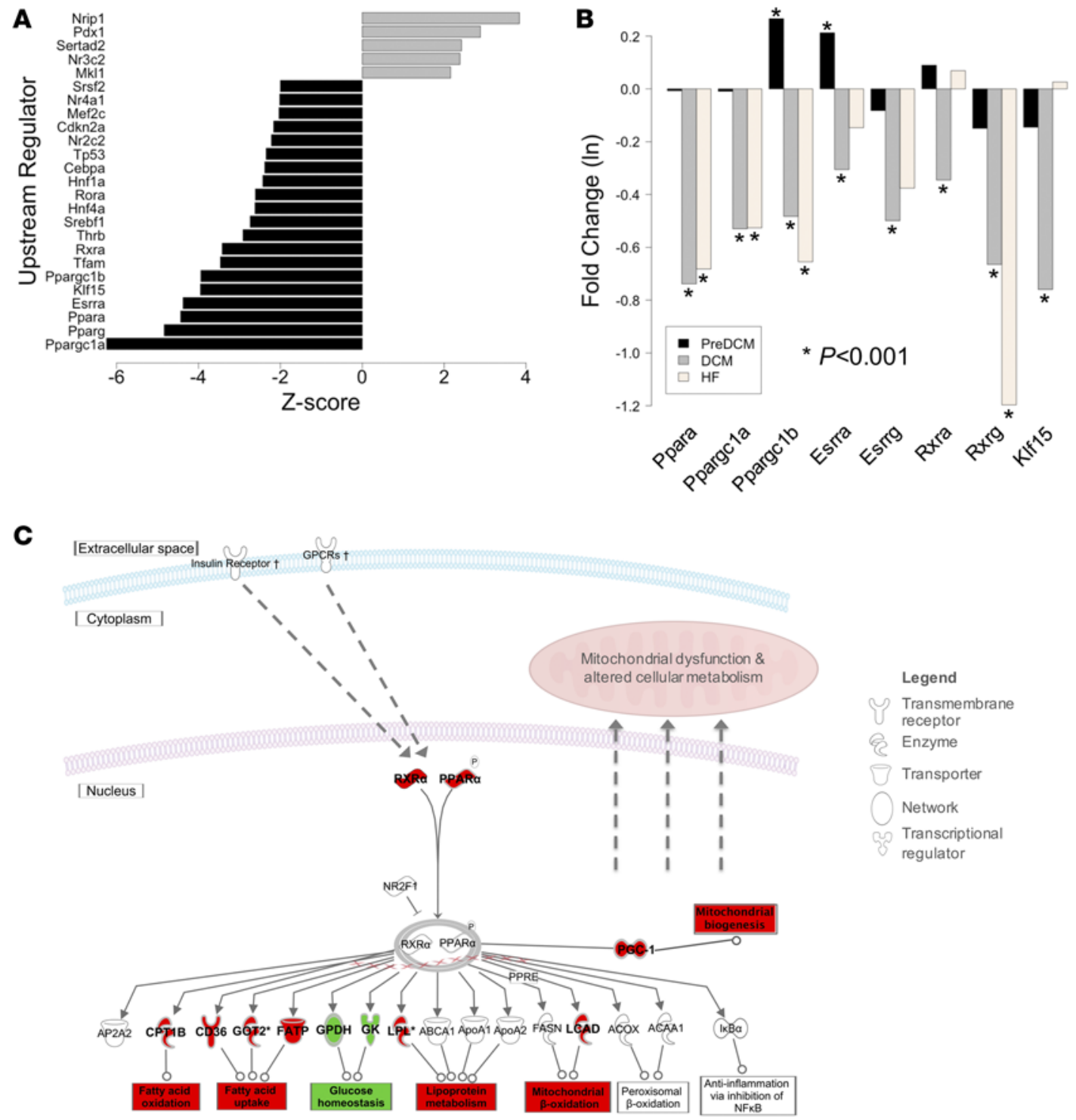

Figure 6. Downregulation of PPAR signaling in PLN ${ }^{\mathrm{RgC} /+}$ with development of DCM. (A) Upstream regulator analysis predicted transcription factors that were activated (gray, Z-score $>2$ ) or inhibited (black, Z-score $<-2$ ) in PLN ${ }^{\mathrm{RgC} /+}$ cardiomyocytes with DCM. (B) PPAR pathway genes and key cofactors were downregulated in PLN ${ }^{R 9 C /+}$ mice with development of DCM $\left.{ }^{*} P<0.001\right)$. Data plotted as natural log (In) of fold-change ( $n=3$ mice pooled prior to RNA-seq). Bayesian $P$ value corrected for multiple hypothesis testing (10). (C) PPAR $\alpha$ and RXR $\alpha$ downregulation is predicted to affect a number of downstream metabolic pathways. Genes and pathways regulated by PPAR $\alpha / R X R \alpha$ that are downregulated (red) or upregulated (green) in PLN ${ }^{R 9 C /+}$ mice with DCM are highlighted. Dashed arrows indicate signaling networks not shown in detail. Adapted from Ingenuity canonical pathway PPAR $\alpha / R X R \alpha$ activation. ${ }^{\dagger}$, representative cell surface receptors that signal to PPAR $\alpha / R X R \alpha$.

$61 \%$ were unique to DCM or HCM; only $28 \%$ of differentially expressed cardiomyocyte genes were shared, while $72 \%$ were unique to either DCM or HCM mice.

To identify potentially distinctive pathways in these cardiomyopathies, we performed additional restricted pathway analyses that included only genes with (i) cardiomyopathy-specific differential expression (e.g., significantly changed only in DCM or HCM), or (ii) cardiomyopathy-selective differential expression (differential expression $>2.5$-fold higher in one cardiomyopathy versus the other). Genes with specific or selective differential expression in nonmyocytes identified both a common and a unique suite of canonical pathways (Supplemental Table 8), transcriptional regulators, growth factors, and kinases (Supplemental Table 9) in DCM and HCM.

Nonmyocytes. Immune signaling pathways were specifically enriched in nonmyocytes from DCM but not HCM mice. Activation of TLR-dependent processes $\left(P=2 \times 10^{-3}\right)$ was inferred from DCM-specific 
A DCM Non-myocyte Pathways

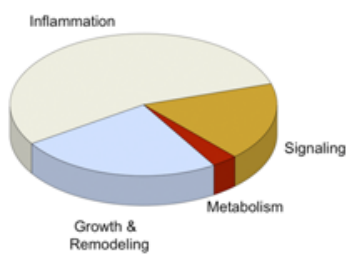

DCM Cardiomyocyte Pathways

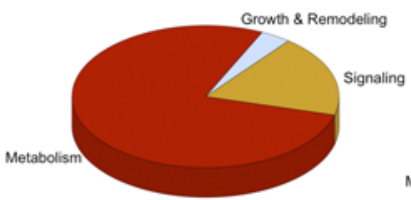

HCM Cardiomyocyte Pathways
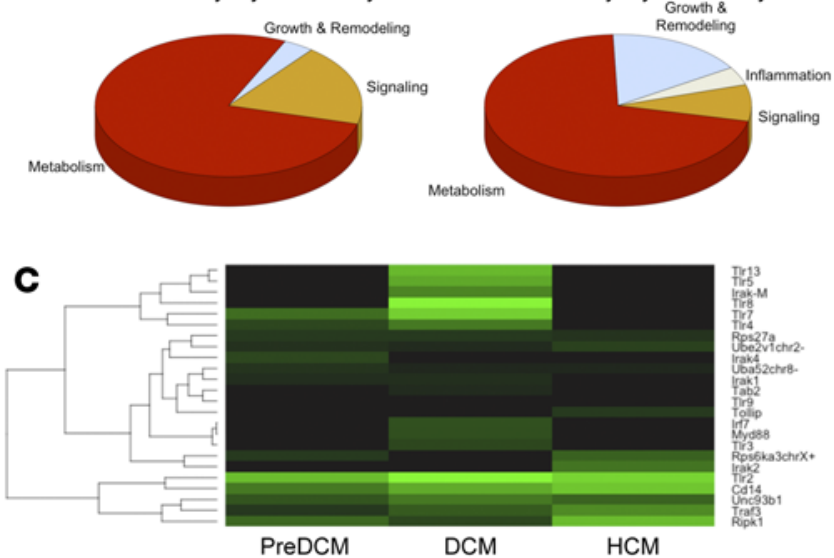

\section{B}

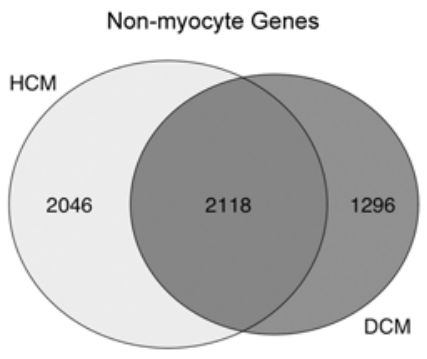

Cardiomyocyte Genes

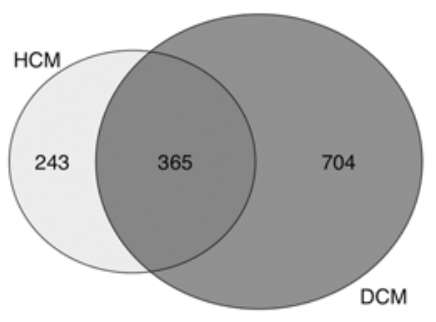

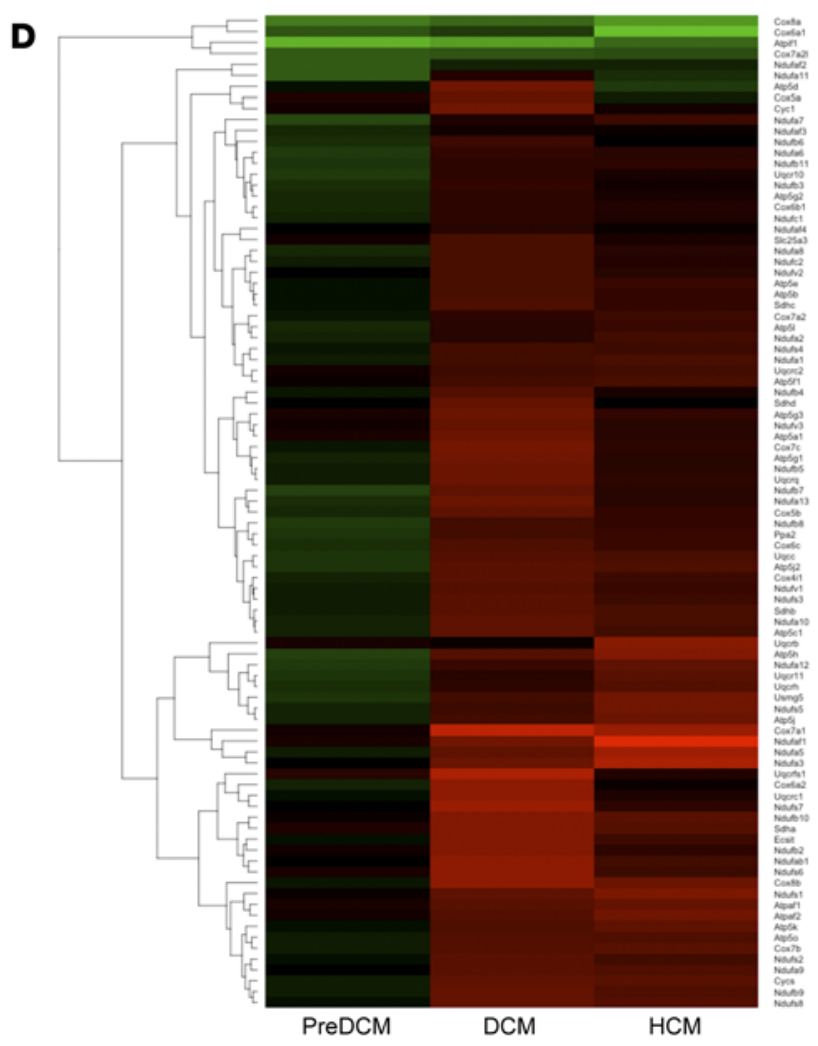
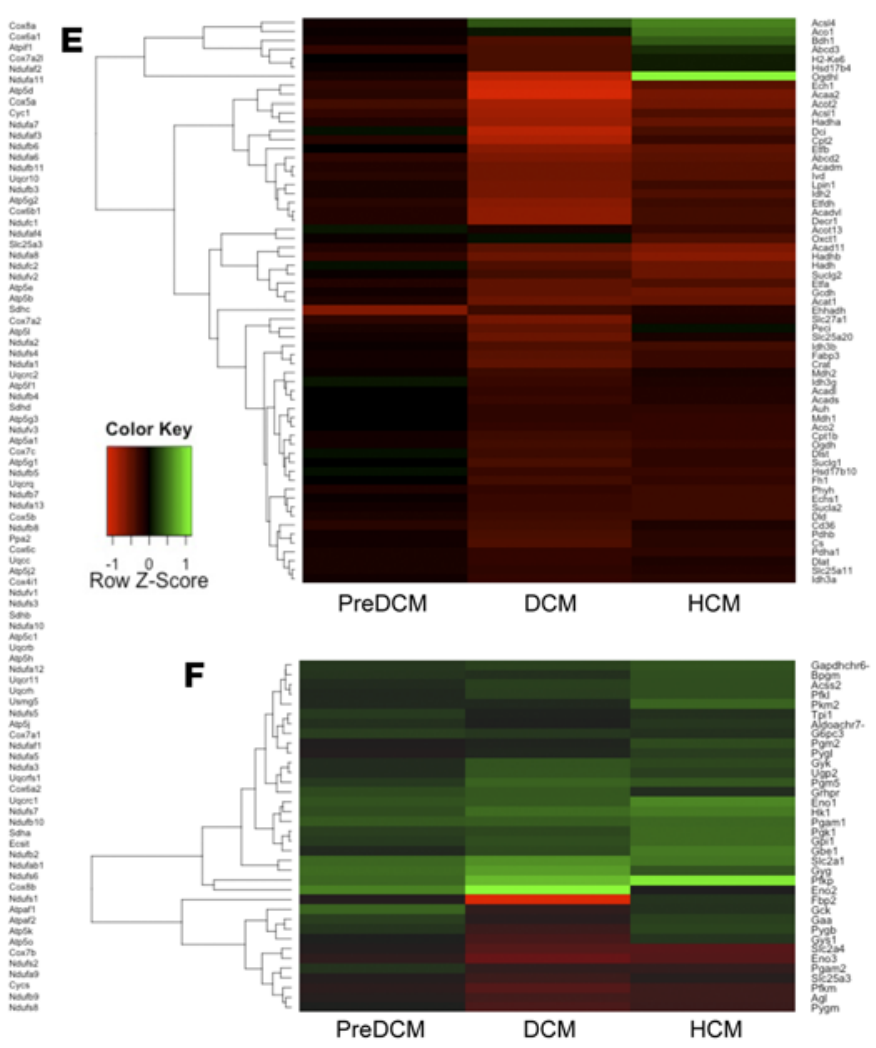

Figure 7. Transcriptional comparisons of DCM and HCM. (A) Enriched (FDR $<0.01$ ) Ingenuity canonical pathways showed a predominance of inflammatory and cell remodeling pathways in nonmyocytes with perturbed cardiomyocyte metabolic pathways in both DCM and HCM. (B) Few differentially expressed genes in nonmyocytes and cardiomyocytes were common to both DCM and HCM. (C) TLR genes were upregulated in DCM but not in HCM. (D) Genes controlling mitochondrial oxidative phosphorylation and (E) those controlling fatty acid oxidation and the citric acid cycle were significantly downregulated, while $(\mathbf{F})$ genes governing glucose metabolism were upregulated with the onset of DCM or HCM. Data plotted in heat maps are fold-change values versus age-matched WT controls ( $n=3$ mice pooled prior to RNA-seq). 
Table 3. Activation of innate immune signaling pathways in DCM but not HCM

\begin{tabular}{|c|c|c|c|}
\hline Pathway & Pre-DCM & DCM & HCM \\
\hline Innate immune response (GO) & $2.4 \times 10^{-13}$ & $4.4 \times 10^{-13}$ & $6.4 \times 10^{-4}$ \\
\hline $\begin{array}{l}\text { Activation of innate immune } \\
\text { response (GO) }\end{array}$ & 0.015 & $2.4 \times 10^{-6}$ & NS \\
\hline $\begin{array}{l}\text { Innate immune response- } \\
\text { activating signal transduction (GO) }\end{array}$ & 0.013 & $9.9 \times 10^{-6}$ & NS \\
\hline $\begin{array}{l}\text { Positive regulation of innate } \\
\text { immune response (GO) }\end{array}$ & 0.030 & $1.3 \times 10^{-5}$ & NS \\
\hline $\begin{array}{l}\text { Negative regulation of innate } \\
\text { immune response (GO) }\end{array}$ & 0.004 & NS & 0.019 \\
\hline TLR signaling pathway (GO) & 0.022 & $3.8 \times 10^{-4}$ & NS \\
\hline TLR signaling pathway (KEGG) & 0.009 & $3.1 \times 10^{-5}$ & 0.014 \\
\hline TLR signaling pathway (IPA) & 0.003 & $7.8 \times 10^{-4}$ & NS \\
\hline
\end{tabular}

upregulation of key TLR genes (Figure 7C and Table 3). DCM nonmyocytes also showed evidence for increased activation of ILs (IL-4 signaling, $P=5 \times 10^{-5}$; IL-8 signaling, $P=4 \times 10^{-4}$; IL-3 signaling, $P=2$ $\left.\times 10^{-3}\right)$ and NFK-B signaling $\left(P=7 \times 10^{-5}\right)$. These genes are generally not expressed in the heart (Supplemental Table 10); thus, pathway enrichment is either reflective of systemic inflammation or overlap with other inflammatory pathways, such as TGF $\beta$. Inferred upstream regulators in DCM nonmyocytes included multiple interferon regulatory factor (IRF) family members and transcription factors involved in innate and adaptive immunity.

By contrast, nonmyocyte genes from HCM hearts predicted activation of a distinct suite of signaling pathways, including noncanonical Wnt signaling (Wnt-calcium signaling, $\left.P=1 \times 10^{-4}\right)$, Her-2 signaling $(P$ $\left.=2 \times 10^{-4}\right)$, and protein kinase A signaling $\left(P=7 \times 10^{-4}\right)$, as well as others. Among the inferred regulators in HCM nonmyocytes are a host of signaling molecules including those governing Wnt signaling (e.g., $W n t 3 A, W n t 5 A$ ), cardiac hypertrophy (e.g., Erbb2, Akt, Src) and the cellular response to hypoxia (Arnt2).

To provide biologic evidence for distinct inflammatory signals in nonmyocytes from DCM and HCM mice, we assessed the effects of the angiotensin II receptor blocker, losartan, in DCM. Our prior studies showed that losartan reduced cardiac fibrosis in $\mathrm{MHC}^{\mathrm{R} 403 \mathrm{O}}$ mice by inhibiting angiotensin II-induced TGF 3 -Smad signaling (27). $\mathrm{PLN}^{\mathrm{R} 9 \mathrm{C} /+}$ mice received continuous losartan treatment from weaning until death with longitudinal echocardiography and terminal assessment of fibrosis. Losartan treatment had no salutary effects on the age at onset of DCM, severity of LV dilation, nor on systolic dysfunction, progression to $\mathrm{HF}$, or age at death. The degree of myocardial fibrosis in treated and untreated PLN ${ }^{\mathrm{R} 9 \mathrm{C} /+}$ mice was identical (Supplemental Figure 5).

Cardiomyocytes. While altered metabolic gene expression was identified in both DCM and HCM, there were some notable differences (Figure 7, D-F). First, though many key PPARs and cofactors were similarly downregulated in HCM (Ppara -2.5-fold, $P=6 \times 10^{-16}$; Ppargcla-1.7-fold, $P=1 \times 10^{-6}$; Esrrg -1.5-fold, $P=0.005 ; R x r g-2.6$-fold, $\left.P=9 \times 10^{-15}\right)$, others were not (Ppargc1b 1.0-fold, $P=0.96$; Esrra 1.0-fold, $P=$ 0.64; Rxra 1.1-fold, $P=0.41$ ), suggesting differences in PPAR signaling between DCM and HCM. Second, a subset of aerobic metabolism genes remained unchanged in HCM (Figure 7E). Analysis of these genes showed enrichment for genes controlling peroxisomal metabolism (Hsd17b4, Hsd17b8/H2-Ke6, Peci, Abcd2, Ehhadh, Abcd3, Acsl4) and consistent with this, pathway analyses showed differential enrichment for peroxisome function between DCM and HCM at baseline (GO term peroxisome [GO:0005777]: DCM $P=4 \times$ $10^{-5}, \mathrm{HCM} P=0.012$; KEGG pathway peroxisome [04146]: DCM $\left.P=2 \times 10^{-5}, \operatorname{HCM} P=0.014\right)$. Finally, Tbx15 was not expressed in HCM; despite this, changes in expression of genes governing glucose metabolism were similar between DCM and HCM (Figure 7F).

Analysis of genes selectively or specifically expressed in either DCM or HCM cardiomyocytes revealed no pathways or upstream regulators that were selectively enriched in HCM. DCM cardiomyocytes showed predicted enrichment for dopamine receptor $\left(P=1 \times 10^{-4}\right)$ and $\beta$-adrenergic $\left(P=3 \times 10^{-4}\right)$ signaling, an observation that is consistent with profound myocardial dysfunction and impending HF. There was 
also evidence for the extensive metabolic changes in DCM cardiomyocytes with predicted inhibition of PPAR-pathway signaling via PGC1 $\alpha$ (Z-score: $-2.9, P=9 \times 10^{-7}$ ) and retinoid X receptor- $\alpha$ (Rxra, Z-score: $-2.6, P=3 \times 10^{-3}$ ), a critical cofactor regulating PPAR $\alpha$ function. In addition, this restricted set of DCM genes predicted inhibition of MED1 (Z-score: $-2.4, P=7 \times 10^{-4}$ ), a subunit of the large TRAP/Mediator transcriptional complex that suppresses gene transcription programs associated with energy expenditure in muscle tissue (28). Notably, while Med1 is predominantly expressed in nonmyocytes at baseline (3.4-fold higher than isolated cardiomyocytes, $P=1 \times 10^{-115}$ ), significant upregulation occurred in isolated DCM cardiomyocytes (1.5-fold, $P=2 \times 10^{-14}$ ). As MED1 inhibition augments mitochondrial biogenesis, (28) this may be a compensatory mechanism in response to energy demands.

\section{Discussion}

Using longitudinal transcriptome analyses, we defined the marked temporal changes in gene expression as $\mathrm{PLN}^{\mathrm{R} 9 \mathrm{C} /+}$ mice transition from preclinical disease to DCM and HF. By parsing gene expression into nonmyocyte and cardiomyocyte compartments, we identified lineage-specific changes. Nonmyocytes in $\mathrm{PLN}^{\mathrm{R9C} /+}$ mice were proliferating and displayed increased transcription of genes that function in innate immunity and inflammation, with notable selective increases in TGF $\beta$ isoforms. Concurrently, cardiomyocytes showed profound dysregulation of cellular metabolism with reduced expression of key PPAR-pathway genes and a marked increase in Tbx15 transcription. By comparison of genes and pathways activated in DCM and HCM hearts, we identified cardiomyopathy-specific gene programs.

Although the $\mathrm{PLN}^{\mathrm{R} 9 \mathrm{C} /+}$ mutation is selectively expressed in cardiomyocytes, transcriptional analyses of nonmyocytes highlighted their early and central role in ventricular remodeling. In pre-DCM hearts, contractile performance was normal, yet we identified increased proliferation of nonmyocytes and altered gene expression, an observation that suggests early dysregulation of calcium homeostasis in mutant cardiomyocytes may promote paracrine signaling to nonmyocytes, possibly via cardiomyocyte-specific expression of cytokines. With the onset of DCM, bioinformatics programs deduced the activation of many inflammatory pathways in nonmyocytes. As genes involved in these pathways (e.g., IRF family members, matricellular proteins, TLRs, etc.) remodel the extracellular matrix of the heart in response to disease, these pathways are likely to increase myocardial fibrosis in DCM and HF (29-31). The TLR pathway was activated in DCM mice and has been implicated in ischemic and nonischemic HF in human patients (30, 32). TLR activation can also promote decreased expression of PGC1 $\alpha$ and PGC1 $\beta$ (33), suggesting a possible link between immune signaling networks and metabolic dysregulation in HF. Notably, the TLR pathway was not activated in HCM, implying different remodeling and profibrosis mechanisms in these cardiomyopathies.

Collagen gene expression was markedly upregulated with progression to DCM and HF, translating into significant extracellular matrix remodeling that ultimately results in massive fibrosis. Stromal remodeling and the synthesis of collagen is a common late-phase step in both wound healing and cancer progression in response to activation of the immune system (34). Thus, immune system activation has overlapping responses between $\mathrm{HF}$ and other fibrotic diseases. However, the systemic inflammatory response observed in $\mathrm{HF}$ is not associated with significant persistence of infiltrating immune cells but rather results in activation of myofibroblasts that play a key role in fibrosis and stromal remodeling (35). Thus, the extensive immune signaling activation observed in cardiac nonmyocytes of $\mathrm{PLN}^{\mathrm{R} 9 \mathrm{C} /+}$ and $\mathrm{MHC}^{403}$ mice suggests that both paracrine and autocrine signaling mechanisms are involved.

We identified activation of canonical Wnt signaling (Wnt/ $\beta$-catenin pathway) in both DCM and HCM. Wnt signaling (both canonical and noncanonical) plays a key role in cardiac development but is largely quiescent in the normal adult heart. However, Wnt signaling is notably altered with various cardiac pathologies including cardiac hypertrophy, myocardial infarction, and HF (25). Importantly, canonical Wnt signaling is necessary for TGF $\beta$-induced fibrosis; hence, it is not surprising that this pathway is activated in both DCM and HCM mice (36). We also identified activation of noncanonical Wnt signaling (Wnt/calcium pathway) only in HCM. The role of noncanonical Wnt signaling pathways in cardiac disease are poorly defined, and it is possible this finding reflects differences in intracellular calcium homeostasis induced by these distinct genetic disorders. But TGF $\beta$ may also activate noncanonical signaling in a context-dependent fashion (37); thus, noncanonical Wnt signaling in HCM mice could be related to differences in TGF $\beta$ signaling, such as may be induced by different TGF $\beta$ isoforms.

Selective activation of TGF $\beta$ family members also distinguished fibrotic pathways in DCM and HCM. Mice with either DCM or HCM have increased expression of Tgfb2 and Tgfb3. However, HCM mice also 
have a significant, 4-fold increase in $T g f b 1$, while this isoform is not significantly elevated in DCM nonmyocytes. Consistent with possible selective activation of TGF $\beta$ isoforms, losartan-mediated inhibition of TGF $\beta 1$ was ineffective in reducing myocardial fibrosis in $\mathrm{PLN}^{\mathrm{R} 9 \mathrm{C} /+}$ mice, although it markedly attenuated fibrosis in HCM hearts (27). Increased expression of another TGF $\beta$ family member, Gdf-15, may also have contributed to the lack of effect of losartan in DCM hearts. Gdf-15 is secreted into the extracellular matrix, where it can translocate into cell nuclei, interrupt Smad complexes, and inhibit TGF $\beta$-dependent Smad signaling pathways (38). Gdf15 was 47-fold increased in DCM $\left(P<1 \times 10^{-300}\right)$ but was not significantly differentially expressed in HCM (2.2-fold, $P=0.005)$, providing further support that profibrotic signaling is distinct in DCM and HCM.

$\mathrm{PLN}^{\mathrm{R} 9 \mathrm{C} /+}$ cardiomyocytes had minimal transcriptional changes until overt DCM was evident. But with onset of morphologic abnormalities, RNA-seq and subsequent qPCR revealed a marked shift in cardiomyocyte metabolic gene levels with decreased expression of genes associated with aerobic respiration and increased expression of those regulating glucose metabolism. Reduced expression of aerobic respiratory genes directly correlates with mitochondrial oxidative capacity in the heart; thus, the overwhelming number of aerobic respiratory genes downregulated with DCM and HF are presumed to be indicative of reduced mitochondrial respiration (39-41). Similarly, expression levels of genes regulating glucose metabolism correlate with metabolic flux $(42,43)$. Thus, we anticipate that the progressive increase in glycolytic gene expression is reflective of increased glucose utilization, possibly through increased nonoxidative glucose metabolism via glycogen synthesis, the hexosamine biosynthetic pathway, and the pentose phosphate shunt. Together, these data support the hypothesis that $\mathrm{HF}$ in $\mathrm{PLN}^{\mathrm{R} 9 \mathrm{C} /+}$ cardiomyocytes is associated with aerobic glycolysis, a phenomenon known as the "Warburg effect" that is common to many forms of cancer (44). Further studies are required to determine the degree of activation of these mechanisms, the usage and role of alternative metabolic substrates, and their collective contribution to DCM progression.

PPAR family member gene expression was depressed with onset of DCM, most notably Ppara, Ppargc1a, and Ppargc1b. PGC1 $\alpha$ is a master regulator of mitochondrial biogenesis and regulates expression of genes governing oxidative phosphorylation in the heart $(45)$. Overexpression $(45,46)$ or ablation of PGC1 $\alpha$ in mice $(39,40)$ causes DCM with diminished cardiac performance and poor stress response - findings that imply tight regulation of PGC1 $\alpha$ is critical to prevent maladaptive responses. PPAR $\alpha$ is a key regulator of myocardial fatty acid oxidation (47), and mice deficient in PPAR $\alpha$ have impaired contractile performance in response to hemodynamic stress $(48,49)$. Expression levels of key PPAR cofactors were also reduced, including RXR $\alpha$ (Rxra), which heterodimerizes with PPAR $\alpha$ to evoke PPAR $\alpha$-induced transcriptional regulation, and estrogen-related receptor $\alpha$ (Esrra), which is induced by and coactivated by PGC1 $\alpha(50,51)$.

Concurrent with the inhibition of PPAR signaling, DCM cardiomyocytes exhibited markedly increased levels of Tbx15. This T-box transcription factor was recently found to regulate the composition of fasttwitch glycolytic fibers in skeletal muscle (26). Ablation of Tbx15 in mice reduced the numbers of glycolytic skeletal muscle fibers and whole body oxygen consumption, and it altered AMPK and IGF signaling. By extrapolation to our data, 8-fold increased levels of Tbx15 in DCM would substantially increase glycolysis in DCM and HF cardiomyocytes. We conclude that the stress-induced reciprocal activation of Tbx15 and attenuation of PPAR signaling accounts for the altered metabolic gene expression that characterized DCM and $\mathrm{HF}$.

While these transcriptional data defined multiple molecules that are excellent candidates for propagating cardiomyopathic pathophysiology, our study has several limitations. Transcript levels do not always correlate with protein levels or activity and are thus an indirect measure of the activation state of transcription factors and regulatory molecules. Hence, these data are associative and would require further testing to perturb molecules and pathways of interest to derive conclusive mechanistic links. Despite normalization of RNA libraries, we recognize that proliferation of nonmyocytes confounds the precise interpretation of increased transcription among nonmyocyte genes. Further, the use of cyclosporine to induce robust hypertrophy in $\mathrm{MHC}^{403}$ mice alters basal expression of some genes and introduces bias when compared with PLN ${ }^{\mathrm{R} 9 \mathrm{C} /+}$, as does the difference in genetic background of these 2 mouse models. Bioinformatics tools also have limitations, as pathway analyses are predicated on previously reported findings that may not be directly relevant to the heart or these cardiomyopathies or may not include newly identified critical genes and mediators of disease. Finally, we recognize the need for extensive additional studies to validate the many new hypotheses generated by these data.

Mindful of these caveats, we conclude that a genetic model of DCM with altered calcium signaling 
incited a myriad of transcriptional changes in the heart that promote myocardial fibrosis and likely metabolic reprogramming. As calcium dysregulation is common to both ischemic and nonischemic HF, we suggest that the genes and pathways activated in $\mathrm{PLN}^{\mathrm{R} 9 \mathrm{C} /+}$ mice define potential therapeutic targets that are relevant to other forms of advanced heart disease. Based on comparative transcriptional analyses of DCM and HCM models, we suggest that these cardiomyopathies share some common pathways but also have distinct molecular programs. Strategies to selectively probe these unique genes and pathways should improve mechanistic insights into pathologic remodeling and clinical phenotypes that characterize DCM and HCM.

\section{Methods}

\section{Mouse models}

Transgenic mice overexpressing $\mathrm{PLN}^{\mathrm{R} 9 \mathrm{C} /+}$ under control of the $\alpha$-cardiac myosin heavy chain promoter have been previously characterized (3). Male $\mathrm{PLN}^{\mathrm{R} 9 \mathrm{C} /+}$ mice were compared with WT male FVB control mice. Mice expressing the $\mathrm{MHC}{ }^{\mathrm{R} 403 \mathrm{O} /+}$ knock-in transgene and comparable age-matched $129 \mathrm{sv} / \mathrm{ev}$ control mice have been previously characterized (9). Hypertrophy was induced in $\mathrm{MHC}^{\mathrm{R} 403 \mathrm{Q} /+}$ mice using oral cyclosporine fed to the mice in the chow as previously described (52).

\section{Echocardiography}

Mice were anesthetized with an isoflurane vaporizer (VetEquip) and attached to ECG leads on a Vevo Mouse Handling Table (VisualSonics Inc.). Chest hair was removed with depilatory cream. Transthoracic echocardiography was performed with heart rate approximately 500-550 bpm using a Vevo 770 High-Resolution In Vivo Micro-Imaging System and RMV 707B scan-head (VisualSonics Inc.). A single, experienced echocardiographer blinded to genotype acquired the images. Parasternal 2D images and M-mode images were acquired to assess LV chamber size and fractional shortening (calculated as FS = [LVEDD-LVESD]/LVEDD).

\section{Quantification of myocardial fibrosis}

Mice were anesthetized with isoflurane, and hearts were exposed by midline thoracotomy. After excision, serial sections of fixed, paraffin-embedded LV — as well as fresh whole mount heart tissue - were stained with Masson trichrome. Fibrosis was quantified as the ratio of fibrotic area to total ventricular area using Image $\mathrm{J}(\mathrm{NIH})$ as previously described (27).

IHC

$\mathrm{PLN}^{\mathrm{R} 9 \mathrm{C} /+}$ mice and age-matched controls were injected i.p. on 3 consecutive days with BrdU (Sigma-Aldrich) at $100 \mathrm{mg} / \mathrm{kg}$ body weight (BW) in PBS at week 8 or week 22. Mice were sacrificed 1 hour after the last injection. Hearts were harvested and then fixed in $4 \%$ paraformaldehyde, were paraffin-embedded, and were sectioned.

Sections were deparaffinized with xylenes and ethanol, rehydrated in deionized water and PBS, and then permeabilized with $0.1 \%$ Tween (BioRad) in PBS. Antigen retrieval was performed by heating slides in target retrieval solution (Dako) at $125^{\circ} \mathrm{C}$ for 30 minutes followed by enzymatic digestion with EcoRI and ExoIII. Sections were blocked in goat serum for 1 hour at room temperature (RT), incubated with antiBrdU antibody (Dako, 1:100) at $4^{\circ} \mathrm{C}$ overnight, washed in PBS, and then incubated with anti-IgG Alexa Fluor 488 (Invitrogen) and anti-wheat germ agglutinin (WGA) Alexa Fluor 594 (Invitrogen, 1:500) at RT for 1 hour. After washing with PBS, sections were incubated for 5 minutes at RT with DAPI (Sigma-Aldrich, 1:10,000), then washed in PBS and mounted using Prolong Gold anti-fade reagent (Invitrogen). Microscopy was performed using a Leica SP2 confocal microscope.

BrdU-labeled cells were counted at $\times 40$ magnification from 4 fields/section per animal; $>13,000$ nuclei were counted per genotype. DAPI-labeled cells were counted at $\times 40$ magnification using Image J. The percent of proliferating cells was calculated as the number of BrdU-labeled cells divided by total DAPI-stained nuclei. Myocyte cell size was measured using Image J. WGA stains highly glycosylated collagen, identifying extracellular matrix, and, therefore, myocyte cell margins. $P$ values were calculated using $\chi^{2}$.

\section{Tissue and cell harvesting for RNA analysis}

Tissue isolation. For collection of whole LV tissue, hearts from PLN ${ }^{\mathrm{R} 9 \mathrm{C} /+}$ mice pre-DCM, with DCM and with overt HF, and age-matched controls were exposed by midline thoracotomy and immediately excised. 
Hearts were bathed in cold $1 \times$ PBS to remove blood and then subjected to left ventriculectomy followed immediately by RNA extraction (below).

Cell isolation. Myocyte and nonmyocyte cell populations were isolated from $\mathrm{PLN}^{\mathrm{R} 9 \mathrm{C} /+}$ mice with echocardiographically confirmed DCM and age-matched controls $(n \geq 6)$. First, hearts were exposed by midline thoracotomy, excised, and placed in cold calcium-free tyrode solution (140 mM NaCl, $5.4 \mathrm{mM} \mathrm{KCl}, 1$

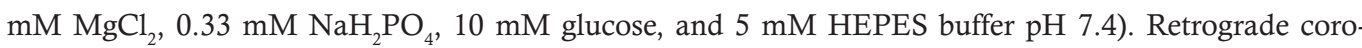
nary perfusion was established via aortic cannulation as described previously (53). The heart was perfused with enzyme buffer (in calcium-free tyrode solution: $0.3 \mathrm{mg} / \mathrm{g}$ BW collagenase D [Roche Diagnostics], 0.4 $\mathrm{mg} / \mathrm{g}$ BW collagenase B [Roche Diagnostics], $0.05 \mathrm{mg} / \mathrm{g}$ BW proteinase XIV [Sigma-Aldrich]) for $15 \mathrm{~min}$ utes. The atria and right ventricle were removed, and the LV was minced into small pieces in transfer buffer (calcium-free tyrode solution $+5 \mathrm{mg} / \mathrm{ml} \mathrm{BSA}$ ) and then passed several times through a sterile pipette. The resulting cell suspension was passed through a mesh filter into a $50 \mathrm{ml}$ centrifuge tube and incubated for 15 minutes at RT to allow myocytes to pellet by gravity. The pellet was serially resuspended every 10 minutes using a calcium gradient (tyrode solution $+5.5 \mathrm{mM}$ glucose $[\mathrm{pH} 7.4]+$ serial calcium concentrations of $0.06 \mathrm{mM}, 0.24 \mathrm{mM}, 0.6 \mathrm{mM}, 1.2 \mathrm{mM}$ ) to derive a cell fraction enriched in myocytes.

The supernatants from the filtered cell solution, as well as the supernatant from the 4 calcium gradient buffers, were pooled and centrifuged at $1,000 \mathrm{~g}$ for 10 minutes at $4^{\circ} \mathrm{C}$. The pellet was resuspended in $\mathrm{DMEM}+10 \% \mathrm{FBS}$, plated on sterile petri dishes, and incubated for 2 hours at $37^{\circ} \mathrm{C}$ in a humidified, $5 \%$ $\mathrm{CO}_{2}$ incubator. Plated cells were washed in sterile PBS to remove debris and nonadherent cells, resulting in a nonmyocyte cell population enriched in cardiac fibroblasts.

$R N A$ isolation. RNA was extracted from excised LV or isolated myocyte and nonmyocyte cell populations using TRIzol (Invitrogen). To minimize biologic variation, RNA was pooled from $n=3$ mice for whole LV tissue samples and $n=6$ mice from isolated cell populations. cDNA libraries were constructed, and 5' RNA-seq was performed as previously described (10). Libraries were sequenced on the Illumina platform and then aligned to the mouse reference sequence $\mathrm{mm} 9$ using Tophat (version 1.4.0). Of note, myosin heavy chain family genes (Myh1-Myh15) were selectively realigned using the STAR aligner because of frequent misalignment with Tophat secondary to the high degree of homology among these genes. The total number of reads of each transcript was normalized to 1 million, with $P$ values of gene fold-changes determined as previously described (10).

$q P C R$. Total RNA $(1 \mu \mathrm{g})$ from excised LV samples was treated with DNase I and then converted to cDNA (SuperScript III First-strand Synthesis System, Invitrogen). Target genes underwent qPCR with SYBR green (Tgfb1, Tgfb2, Tgfb3, Gdf15) on a 7500 Fast RT-PCR System (Applied Biosystems) or TAQman probes (Ppara, Ppargc1a, Ppargc1b, Tbx15, Ndufs7, Cox7a1, Atp5a1, Polr3h) using a BioRad QX200 Droplet Digital PCR System. Gapdh was progressively upregulated in PLN ${ }^{\mathrm{R} 9 \mathrm{C} /+}$ mice (pre-DCM, 1.2-fold, $P$ $=1 \times 10^{-5} ; \mathrm{DCM}$, 1.3-fold, $P=2 \times 10^{-10} ; \mathrm{HF}, 1.6$-fold, $P=2 \times 10^{-28}$ ). Hence, Polr3h was chosen as a control for normalization because of robust cardiac expression and minimal biologic variability between $\mathrm{PLN}^{\mathrm{R} 9 \mathrm{C} /+}$ and WT mice at all time points. See Supplemental Table 11 for probe and primer details.

Bioinformatics pipeline for pathway analysis. We defined genes as being differentially expressed if they met the following 3 criteria: (i) normalized read count $>2$ copies/million reads in the RNA-seq library, (ii) fold-change $>1.33$ (upregulated) or $<-1.33$ (downregulated), and (iii) $P<0.001$ for the comparison of normalized read counts between mutant and control animals. Our bioinformatics pipeline is described in Supplemental Figure 4 and was executed using R (version 3.0.1). Culled lists representing those genes differentially expressed in nonmyocytes or myocytes were then subjected to pathway analysis using 2 bioinformatics platforms: IPA (Qiagen) and GO-seq (Bioconductor package, "Goseq”).

$I P A$. IPA uses proprietary algorithms and a proprietary database of gene information to generate a number of different analyses based on a set of user-identified genes (http://www.ingenuity.com/products/ ipa). We used IPA to identify enriched canonical pathways and to predict possible upstream regulatory molecules from our RNA-seq data.

Only genes identified as differentially expressed (Supplemental Figure 4) were uploaded into IPA. We used the following IPA core analysis settings: general settings, reference set = Ingenuity knowledge base (genes only), relationships to consider $=$ direct and indirect relationships; data sources $=$ all; confidence $=$ experimentally observed only; species $=$ all. Some gene symbols in our $\mathrm{mm} 9$ database were not identifiable by IPA and were consequently not used in the analysis (range 12\%-19\% for different datasets).

We first reviewed Ingenuity canonical pathways to study known biological pathways and processes that 
were enriched in our differentially expressed genes. Specific canonical pathways of interest were assessed for significance based on the nominal $P$ value as determined by Ingenuity software, which is ascertained by a right-tailed Fisher exact test. This $P$ value is a measure of the likelihood that the association between a particular canonical pathway and our differentially expressed genes is due to chance. A nominal $P$ value of $<0.05$ was considered significant, when considering specific pathways of interest. To control for the rate of false discoveries when analyzing all significantly enriched pathways for a given set of genes, significance was based on the $P$ value corrected for multiple hypothesis testing (Benjamini-Hochberg). In this case, the $P$ value represents the FDR. We used a FDR $<0.01$ as significant. The canonical pathways were generated through the use of QIAGEN's IPA (http://www.qiagen.com/ingenuity). For more details on calculation of $P$ values by IPA, the interested reader is referred to the white paper, "Calculating and Interpreting the $P$ values for Functions, Pathways and Lists in IPA" (http://www.ingenuity.com/wp-content/themes/ingenuity-qiagen/pdf/ipa/functions-pathways-pval-whitepaper.pdf).

We also used the IPA upstream regulator tool. This attempts to predict the functional status of molecules (upstream regulators) such as transcription factors, kinases, and growth factors based on known downstream targets (the input set of differentially expressed genes). Two metrics of significance were used. The overlap $P$ value represents the significance in the overlap between the identified differentially expressed genes (the known downstream targets) and all genes associated with a particular upstream regulator in the Ingenuity database. We used a $P$ value threshold of $<0.01$ as significant. The Z-score also accounts for the direction of activation of the differentially expressed genes in relation to published literature with respect to a particular upstream regulator, thereby allowing a prediction of whether the upstream regulator is activated or inhibited. Four possibilities exist: (i) a gene known to be activated by an upstream regulator that is upregulated in the dataset predicts activation of the upstream regulator; (ii) a gene known to be activated by an upstream regulator that is downregulated in the dataset predicts inhibition of the upstream regulator; (iii) a gene known to be inhibited by an upstream regulator that is upregulated in the dataset predicts inhibition of the upstream regulator; and (iv) a gene known to be inhibited by an upstream regulator that is downregulated in the dataset predicts activation of the upstream regulator. The Z-score accounts for both the total number of genes differentially expressed and the direction of activation; a Z-score $\geq 2$ (activated) or $\leq-2$ (inhibited) was considered significant. Additional details can be found on the IPA website (http://pages.ingenuity.com/rs/ingenuity/images/0812 upstream_regulator_analysis_whitepaper.pdf).

Goseq. Goseq is a free, R-software-based program that allows for identification of GO terms and KEGG pathways from a set of user-identified genes set against a customizable, user-identified background dataset as described previously (54). R packages Goseq (v. 1.12.0), GO.db (v. 2.9.0), and org.Mm.eg.db (v. 2.9.0) were used. In brief, differentially expressed genes were identified within a vector of all genes expressed in LV tissue (normalized read count > 2 copies/million reads in either WT or PLN ${ }^{\mathrm{R} 9 \mathrm{C} /+}$ RNA-seq libraries), which served as the background dataset, at a given stage of disease. Goseq was then used to identify significantly enriched GO terms and KEGG pathways. $P$ values were adjusted for multiple hypothesis testing (Bonferroni) and a FDR $<0.05$ was considered significant.

\section{Statistics}

Data are presented as mean \pm SD. Between groups, differences were calculated using a 2-tailed Student's $t$ test or a Bayesian $P$ value as described previously for RNA-seq data (10). Statistical significance for pathway analysis tools were calculated as described in the above referenced links; $P$ values were corrected for multiple hypothesis testing where appropriate and are represented as the FDR. $P$ values $<0.05$ were considered significant except as noted ( $P<0.001$ for RNA-seq expression levels, FDR $<0.01$ for IPA canonical pathways).

\section{Study approval}

Studies were approved by the Animal Care and Use Committee of Harvard Medical School. All care and procedures were performed as per these guidelines.

\section{Author contributions}

$\mathrm{MAB}$ designed analyses, conducted experiments, acquired and analyzed data, and drafted the manuscript. SC designed analyses, conducted experiments, and acquired and analyzed data. HW conducted experiments and analyzed data. JMG and DCC conducted experiments. DAC provided reagents and assisted 
with animal care. MGP and SRD processed and analyzed data. SE and TK assisted with experimental design and execution. JGS and CES designed analyses, analyzed data, and drafted the manuscript.

\section{Acknowledgments}

This work was supported in part by the Clinical Skills Development Core Training Grant (NHLBI U10HL110337; to M.A. Burke), the John S. LaDue Memorial Fellowship in Cardiology at Harvard Medical School (to M.A. Burke), the NIH (R01 HL080494-08, R01 HL084553-06A1, to J.G. Seidman) the Howard Hughes Medical Institute (to C.E. Seidman), and the LeDucq Foundation (to J.G. Seidman and C.E. Seidman).

Address correspondence to: Michael A. Burke, WMB 322, Cardiology Division, Emory University School of Medicine, 101 Woodruff Circle, Atlanta, Georgia 30322, USA. Phone: 404.712.2690; E-mail: michael.burke@emory.edu. Or to: Christine E. Seidman, NRB 256, Department of Genetics, Harvard Medical School, 77 Ave Louis Pasteur, Boston, Massachusetts 02115, USA. Phone: 617.432.7871; E-mail: cseidman@genetics.med.havard.edu.

M.A. Burke's present address is: Cardiology Division, Department of Medicine, Emory University School of Medicine, Atlanta, Georgia, USA.

1. Hershberger RE, Hedges DJ, Morales A. Dilated cardiomyopathy: the complexity of a diverse genetic architecture. Nat Rev Cardiol. 2013;10(9):531-547.

2. Gorski PA, Ceholski DK, Hajjar RJ. Altered myocardial calcium cycling and energetics in heart failure-a rational approach for disease treatment. Cell Metab. 2015;21(2):183-194.

3. Schmitt JP, et al. Dilated cardiomyopathy and heart failure caused by a mutation in phospholamban. Science. 2003;299(5611):1410-1413.

4. Truszkowska GT, et al. A study in Polish patients with cardiomyopathy emphasizes pathogenicity of phospholamban (PLN) mutations at amino acid position 9 and low penetrance of heterozygous null PLN mutations. BMC Med Genet. 2015;16:21.

5. Fish M, et al. Mutation analysis of the phospholamban gene in 315 South Africans with dilated, hypertrophic, peripartum and arrhythmogenic right ventricular cardiomyopathies. Sci Rep. 2016;6:22235.

6. Maron BJ, Casey SA, Poliac LC, Gohman TE, Almquist AK, Aeppli DM. Clinical course of hypertrophic cardiomyopathy in a regional United States cohort. JAMA. 1999;281(7):650-655.

7. Thaman R, et al. Prevalence and clinical significance of systolic impairment in hypertrophic cardiomyopathy. Heart 2005;91(7):920-925.

8. Harris KM, et al. Prevalence, clinical profile, and significance of left ventricular remodeling in the end-stage phase of hypertrophic cardiomyopathy. Circulation. 2006;114(3):216-225.

9. Geisterfer-Lowrance AA, et al. A mouse model of familial hypertrophic cardiomyopathy. Science. 1996;272(5262):731-734.

10. Christodoulou DC, et al. 5'RNA-Seq identifies Fhl1 as a genetic modifier in cardiomyopathy. J Clin Invest. 2014;124(3):13641370 .

11. Cucoranu I, et al. NAD(P)H oxidase 4 mediates transforming growth factor- $\beta 1$-induced differentiation of cardiac fibroblasts into myofibroblasts. Circ Res. 2005;97(9):900-907.

12. Kim W, et al. RUNX1 is essential for mesenchymal stem cell proliferation and myofibroblast differentiation. Proc Natl Acad Sci U $S$ A. 2014;111(46):16389-16394.

13. Raaz U, et al. Transcription factor Runx2 promotes aortic fibrosis and stiffness in type 2 diabetes mellitus. Circ Res. 2015;117(6):513-524.

14. Mullen AC, et al. Master transcription factors determine cell-type-specific responses to TGF- $\beta$ signaling. Cell. 2011;147(3):565576.

15. Braitsch CM, Kanisicak O, van Berlo JH, Molkentin JD, Yutzey KE. Differential expression of embryonic epicardial progenitor markers and localization of cardiac fibrosis in adult ischemic injury and hypertensive heart disease. J Mol Cell Cardiol. 2013;65:108-119.

16. Sazonova O, et al. Characterization of TCF21 downstream target regions identifies a transcriptional network linking multiple independent coronary artery disease loci. PLoS Genet. 2015;11(5):e1005202.

17. Ponticos M, Harvey C, Ikeda T, Abraham D, Bou-Gharios G. JunB mediates enhancer/promoter activity of COL1A2 following TGF- $\beta$ induction. Nucleic Acids Res. 2009;37(16):5378-5389.

18. Perry MC, et al. ERBB2 deficiency alters an E2F-1-dependent adaptive stress response and leads to cardiac dysfunction. Mol Cell Biol. 2014;34(23):4232-4243.

19. Faherty N, et al. TGF $\beta$ and CCN2/CTGF mediate actin related gene expression by differential E2F1/CREB activation. BMC Genomics. 2013;14:525.

20. Zhang Y, et al. E2F1 is a novel fibrogenic gene that regulates cholestatic liver fibrosis through the Egr-1/SHP/EID1 network. Hepatology. 2014;60(3):919-930.

21. Brooks AC, DeMartino AM, Brainard RE, Brittian KR, Bhatnagar A, Jones SP. Induction of activating transcription factor 3 limits survival following infarct-induced heart failure in mice. Am J Physiol Heart Circ Physiol. 2015;309(8):H1326-H1335.

22. Koren L, et al. ATF3-dependent cross-talk between cardiomyocytes and macrophages promotes cardiac maladaptive remodel- 
ing. Int J Cardiol. 2015;198:232-240.

23. Liu Y, et al. Sox17 is essential for the specification of cardiac mesoderm in embryonic stem cells. Proc Natl Acad Sci U S A. 2007;104(10):3859-3864.

24. Singh R, et al. Tbx20 interacts with smads to confine tbx2 expression to the atrioventricular canal. Circ Res. 2009;105(5):442452.

25. Dawson K, Aflaki M, Nattel S. Role of the Wnt-Frizzled system in cardiac pathophysiology: a rapidly developing, poorly understood area with enormous potential. J Physiol. 2013;591(6):1409-1432.

26. Lee KY, et al. Tbx15 controls skeletal muscle fibre-type determination and muscle metabolism. Nat Commun. 2015;6:8054.

27. Teekakirikul $\mathrm{P}$, et al. Cardiac fibrosis in mice with hypertrophic cardiomyopathy is mediated by non-myocyte proliferation and requires Tgf- $\beta$. J Clin Invest. 2010;120(10):3520-3529.

28. Chen W, Zhang X, Birsoy K, Roeder RG. A muscle-specific knockout implicates nuclear receptor coactivator MED1 in the regulation of glucose and energy metabolism. Proc Natl Acad Sci U S A. 2010;107(22):10196-10201.

29. Yaxing Z, Xiao-Jing Z, Hongliang L. Targeting interferon regulatory factor for cardiometabolic diseases: opportunities and challenges [published online ahead of print August 4, 2015]. Curr Drug Targets. PMID: 26240052

30. Mann DL, Topkara VK, Evans S, Barger PM. Innate immunity in the adult mammalian heart: for whom the cell tolls. Trans Am Clin Climatol Assoc. 2010;121:34-50.

31. Okamoto H, Imanaka-Yoshida K. Matricellular proteins: new molecular targets to prevent heart failure. Cardiovasc Ther. 2012;30(4):e198-209.

32. Frantz S, et al. Toll4 (TLR4) expression in cardiac myocytes in normal and failing myocardium. J Clin Invest. 1999;104(3):271280

33. Schilling J, Lai L, Sambandam N, Dey CE, Leone TC, Kelly DP. Toll-like receptor-mediated inflammatory signaling reprograms cardiac energy metabolism by repressing peroxisome proliferator-activated receptor $\gamma$ coactivator- 1 signaling. Circ Heart Fail. 2011;4(4):474-482.

34. Dvorak HF. Tumors: wounds that do not heal-redux. Cancer Immunol Res. 2015;3(1):1-11.

35. Weber KT, Sun Y, Bhattacharya SK, Ahokas RA, Gerling IC. Myofibroblast-mediated mechanisms of pathological remodelling of the heart. Nat Rev Cardiol. 2013;10(1):15-26.

36. Akhmetshina A, et al. Activation of canonical Wnt signalling is required for TGF- $\beta$-mediated fibrosis. Nat Commun. 2012;3:735

37. Chopra S, Kumar N, Rangarajan A, Kondaiah P. Context dependent non canonical WNT signaling mediates activation of fibroblasts by transforming growth factor- $\beta$. Exp Cell Res. 2015;334(2):246-259.

38. Min KW, et al. NAG-1/GDF15 accumulates in the nucleus and modulates transcriptional regulation of the Smad pathway. Oncogene. 2016;35(3):377-388.

39. Arany Z, et al. Transcriptional coactivator PGC-1 $\alpha$ controls the energy state and contractile function of cardiac muscle. Cell Metab. 2005;1(4):259-271.

40. Arany Z, Novikov M, Chin S, Ma Y, Rosenzweig A, Spiegelman BM. Transverse aortic constriction leads to accelerated heart failure in mice lacking PPAR- $\gamma$ coactivator 1 $\alpha$. Proc Natl Acad Sci U S A. 2006;103(26):10086-10091.

41. Puente BN, et al. The oxygen-rich postnatal environment induces cardiomyocyte cell-cycle arrest through DNA damage response. Cell. 2014;157(3):565-579.

42. Carley AN, Taglieri DM, Bi J, Solaro RJ, Lewandowski ED. Metabolic efficiency promotes protection from pressure overload in hearts expressing slow skeletal troponin I. Circ Heart Fail. 2015;8(1):119-127.

43. Wang ZV, et al. Spliced X-box binding protein 1 couples the unfolded protein response to hexosamine biosynthetic pathway. Cell. 2014;156(6):1179-1192

44. Vander Heiden MG, Cantley LC, Thompson CB. Understanding the Warburg effect: the metabolic requirements of cell proliferation. Science. 2009;324(5930):1029-1033.

45. Lehman JJ, Barger PM, Kovacs A, Saffitz JE, Medeiros DM, Kelly DP. Peroxisome proliferator-activated receptor $\gamma$ coactivator-1 promotes cardiac mitochondrial biogenesis. J Clin Invest. 2000;106(7):847-856.

46. Russell LK, et al. Cardiac-specific induction of the transcriptional coactivator peroxisome proliferator-activated receptor $\gamma$ coactivator-1 $\alpha$ promotes mitochondrial biogenesis and reversible cardiomyopathy in a developmental stage-dependent manner. Circ Res. 2004;94(4):525-533.

47. Djouadi F, et al. A gender-related defect in lipid metabolism and glucose homeostasis in peroxisome proliferator- activated receptor $\alpha$ - deficient mice. J Clin Invest. 1998;102(6):1083-1091.

48. Luptak I, Balschi JA, Xing Y, Leone TC, Kelly DP, Tian R. Decreased contractile and metabolic reserve in peroxisome proliferator-activated receptor- $\alpha$-null hearts can be rescued by increasing glucose transport and utilization. Circulation 2005;112(15):2339-46

49. Smeets PJ, et al. Cardiac hypertrophy is enhanced in PPAR $\alpha^{-/-}$mice in response to chronic pressure overload. Cardiovasc Res. 2008;78(1):79-89

50. Brown JD, Plutzky J. Peroxisome proliferator-activated receptors as transcriptional nodal points and therapeutic targets. Circulation. 2007;115(4):518-533.

51. Villena JA, Kralli A. ERR $\alpha$ : a metabolic function for the oldest orphan. Trends Endocrinol Metab. 2008;19(8):269-276.

52. Fatkin D, et al. An abnormal $\mathrm{Ca}^{2+}$ response in mutant sarcomere protein-mediated familial hypertrophic cardiomyopathy. $J C l i n$ Invest. 2000;106(11):1351-1359.

53. Liao R, Jain M. Isolation, culture, and functional analysis of adult mouse cardiomyocytes. Methods Mol Med. 2007;139:251-262

54. Young MD, Wakefield MJ, Smyth GK, Oshlack A. Gene ontology analysis for RNA-seq: accounting for selection bias. Genome Biol. 2010;11(2):R14. 